\title{
A!
}

This is an electronic reprint of the original article.

This reprint may differ from the original in pagination and typographic detail.

Julku, Aleksi; Bruun, Georg M.; Törmä, Päivi

\section{Excitations of a Bose-Einstein condensate and the quantum geometry of a flat band}

Published in:

Physical Review B

DOI:

10.1103/PhysRevB.104.144507

Published: 21/10/2021

Document Version

Publisher's PDF, also known as Version of record

Please cite the original version:

Julku, A., Bruun, G. M., \& Törmä, P. (2021). Excitations of a Bose-Einstein condensate and the quantum geometry of a flat band. Physical Review B, 104(14), [A33]. https://doi.org/10.1103/PhysRevB.104.144507

This material is protected by copyright and other intellectual property rights, and duplication or sale of all or part of any of the repository collections is not permitted, except that material may be duplicated by you for your research use or educational purposes in electronic or print form. You must obtain permission for any other use. Electronic or print copies may not be offered, whether for sale or otherwise to anyone who is not an authorised user. 


\title{
Excitations of a Bose-Einstein condensate and the quantum geometry of a flat band
}

\author{
Aleksi Julku $\odot, 1,2,{ }^{*}$ Georg M. Bruun $\odot{ }^{2,3}$ and Päivi Törmä $\odot^{1, \dagger}$ \\ ${ }^{1}$ Department of Applied Physics, Aalto University, P.O. Box 15100, 00076 Aalto, Finland \\ ${ }^{2}$ Center for Complex Quantum Systems, Department of Physics and Astronomy, \\ Aarhus University, Ny Munkegade 120, DK-8000 Aarhus C, Denmark \\ ${ }^{3}$ Shenzhen Institute for Quantum Science and Engineering and Department of Physics, \\ Southern University of Science and Technology, Shenzhen 518055, China
}

(Received 2 June 2021; revised 1 September 2021; accepted 9 September 2021; published 21 October 2021)

\begin{abstract}
The quantum geometry of Bloch states fundamentally affects a wide range of physical phenomena. The quantum Hall effect, for example, is governed by the Chern number, and flat-band superconductivity by the distance between the Bloch states: the quantum metric. While understanding quantum geometry phenomena in the context of fermions is well established, less is known about the role of quantum geometry in bosonic systems where particles can undergo Bose-Einstein condensation (BEC). In conventional single-band or continuum systems, excitations of a weakly interacting BEC are determined by the condensate density and the interparticle interaction energy. In contrast to this, we discover here fundamental connections between the properties of a weakly interacting BEC and the underlying quantum geometry of a multiband lattice system. We show that, in the flat-band limit, the defining physical quantities of BEC, namely, the speed of sound and the quantum depletion, are dictated solely by the quantum geometry. We find that the speed of sound becomes proportional to the quantum metric of the condensed state. Furthermore, the quantum distance between the Bloch functions forces the quantum depletion and the quantum fluctuations of the density-density correlation to obtain finite values for infinitesimally small interactions. This is in striking contrast to dispersive bands where these quantities vanish with the interaction strength. Additionally, we show how in the flat-band limit the supercurrent is carried by the quantum fluctuations and is determined by the Berry connections of the Bloch states. Our results reveal how nontrivial quantum geometry allows reaching strong quantum correlation regime of condensed bosons even with weak interactions. This is highly relevant, for example, for polariton and photon BECs where interparticle interactions are inherently small. Our predictions can be experimentally tested with flat-band lattices already implemented in ultracold gases and various photonic platforms.
\end{abstract}

DOI: 10.1103/PhysRevB.104.144507

\section{INTRODUCTION}

In recent years, it has become clear that the quantum geometry of Bloch states is a fundamentally important property that complements the information given by energy band dispersions. If the unit cell hosts multiple orbitals (lattice sites, spins, etc.), the Bloch states become vectors in the orbital basis. Consequently, the quantum geometry of the band, namely, the phase and amplitude distances of the Bloch states, may become nontrivial. In other words, they may differ drastically from the single-band or continuum systems. This is quantified by the quantum geometric tensor [1] whose imaginary part is the Berry curvature and real part is the quantum metric, a measure of distance between two quantum states [2].

Quantum geometry can play a central role in determining the properties of a given system. For instance, Berry curvature governs the anomalous transport of an electron wave packet [3], while its integral over the Brillouin zone (BZ) gives the Chern number, which, among other topological invariants, is

\footnotetext{
*Corresponding author: aleksi.julku@gmail.com

${ }^{\dagger}$ Corresponding author: paivi.torma@aalto.fi
}

central in explaining the quantum Hall effect and topological insulators [4-9]. The importance of the quantum metric for phenomena such as superconductivity [10-13], orbital magnetic susceptibility [14,15], and light-matter coupling [16] has been understood only recently, and the interest in the quantum metric is rapidly growing [17-20]. Quantum geometric concepts have also been proposed for bosonic systems composed of light, bosonic atoms, or collective excitations [21-30].

While prominent topological properties can already arise from single-particle physics, interactions between particles lead to even more intriguing phenomena [7,31-37] such as topological superconductors and fractional Chern insulators. In the context of interacting systems, (nearly) dispersionless bands with diverging effective mass, so-called flat bands [38], are particularly exciting for two reasons. First, quantum manybody interaction and correlation effects are expected to be strong when the kinetic energy scale is quenched. Second, the effects of the Bloch state quantum geometry are likely to dominate if the band dispersion itself is featureless. Superconductivity is an important example of this, as the critical temperature is predicted to be exponentially enhanced in a flat band [39], and the stability of a supercurrent guaranteed by a nonzero quantum metric and the Chern number 
[10-12]. Flat-band systems, both for fermions and bosons, can be experimentally realized, for example, in ultracold gases, photonic and polaritonic systems, and atomistic designer matter [38,40-46]. A well-known example of a nearly flat-band system is given by twisted bilayer graphene, where the observed superconductivity [47-49] has indeed been proposed to be influenced by quantum geometry [17-20].

While geometric properties of quantum states in fermionic systems are widely explored, the significance of quantum geometry in the context of bosonic systems remains less studied. It is therefore natural to ask how the quantum geometry affects the Bose-Einstein condensation (BEC) of interacting bosons, particularly in a flat band. There are obvious outstanding puzzles. Since the states of the flat band are degenerate, which one is chosen for BEC? Since single particles are localized in a flat band, is superfluidity even possible? Due to the high degeneracy, is the condensate immediately fragmented? It has been theoretically shown that interactions between the bosons enable mass current [50-55], even in a flat band, and thereby a BEC [56]. Semiconductor polariton condensates have been experimentally studied in Lieb [57-60] and kagome [61] lattices, showing fragmentation and localization. Theory work on the kagome lattice predicts that due to interactions, a certain lattice momentum is favorable for BEC, even when initially all momenta have degenerate energies [62]. Thus far, studies of flat-band BECs have mainly focused on mean-field properties, although Ref. [62] analyzed the stability of the condensate against quantum fluctuations using Bogoliubov theory. However, the density-density response and excitation density have not been calculated and, importantly, the relation between the quantum geometry and the excitations of a BEC has not been considered.

Here, we investigate excitations of weakly interacting bosonic condensates and reveal fundamental connections to quantum geometry. In a flat band, we find condensate behavior radically different from that in a dispersive band. By applying multiband Bogoliubov theory, we study the speed of sound $c_{s}$, density-density correlations, superfluid weight $D^{s}$, and excitation density $n_{\mathrm{ex}}$, i.e., the amount of particles depleted from the condensate due to interactions (so-called quantum depletion).

In a flat band, $c_{s}$ is found to be determined by the quantum metric of the condensate state such that a finite quantum metric guarantees a finite $c_{s}$. Moreover, in contrast to the usual square-root dependence [63], $c_{s}$ in a flat band is linearly proportional to the interaction because the quantum metric provides an interaction-dependent effective mass.

We furthermore show that the excitation density $n_{\mathrm{ex}}$ is found to behave in a striking way in a flat band: it has a finite value for both finite and infinitesimally small interactions. This is remarkable for two reasons. First, it is totally different from the conventional dispersive case where $n_{\mathrm{ex}}$ vanishes with the interactions. Second, for finite interactions, one could intuitively expect the excitation fraction to diverge in a band with flat energy spectrum, as the excitations have no kinetic energy cost. However, it turns out that the excitation density for small interactions is determined by the quantum distance between the condensed state and the other states of the band: a finite distance between them curtails the excitation fraction from diverging. A salient point here is that the excitation density does not depend on the total density. Therefore, by decreasing the condensation density, one can increase the depletion of the condensate, even in the $U \rightarrow 0$ limit. In this way, the importance of quantum fluctuations and correlations can be significantly enhanced at the weak-coupling limit.

Density-density correlations are typically dominated by the macroscopic population of the condensate. We show that in a flat band the quantum correlations between excitations are also prominent. In fact, the quantum fluctuation contribution obtains a finite value, even in the $U \rightarrow 0$ limit, due to quantum geometric properties of the flat band, similar to $n_{\mathrm{ex}}$. This means that in flat-band BECs, quantum geometry can provide access to manifestly quantum (beyond mean-field) correlations, even for weak interactions.

Finally, the superfluid weight is usually given by the density of the condensed bosons and the band dispersion relation. In a flat band, we find that finite superfluidity arises extraordinarily due to the quantum fluctuations only and is determined by the Berry connections of the Bloch states. We prove this analytically in the noninteracting limit and show that for the kagome lattice the result is valid for a wide range of interaction values.

To summarize, in contrast to a dispersive band BEC, the excitation properties of a weakly interacting flat-band $\mathrm{BEC}$ depend on the underlying quantum geometry in the following way. (1) The speed of sound $c_{s}$ is given by the quantum metric and depends linearly (in contrast to the conventional square-root dependence) on the interaction strength. (2) The excitation density can be nonzero even in the limit $U \rightarrow 0$ and is given by the quantum distance among the flat-band states. (3) The quantum fluctuations can be significant even for $U \rightarrow 0$ and are determined by the quantum distance. (4) The superfluid weight is induced by the quantum fluctuations and has a quantum geometric origin. Our main results are summarized in Fig. 1.

Our present work accompanies the joint study of Ref. [64] and together these two works establish fundamental relations between the quantum geometry and BEC. We show that bosonic condensation and superfluidity can be stable in a flat band if there is a finite quantum metric and a quantum distance between the condensed state and noncondensed states of the band. Importantly, the fluctuations dominate over mean-field properties in several physical observables, making flat bands a promising platform for realizing strongly correlated bosonic systems, even at the weak interaction limit.

\section{THEORETICAL FRAMEWORK OF BEC AND BOGOLIUBOV APPROXIMATION IN A FLAT-BAND SYSTEM}

We consider a weakly interacting BEC in a multiband system described by the Bose-Hubbard Hamiltonian

$$
\begin{aligned}
H= & \sum_{i \alpha, j \beta} c_{i \alpha}^{\dagger} \mathcal{H}_{i \alpha, j \beta} c_{j \beta}-\mu \sum_{i \alpha} c_{i \alpha}^{\dagger} c_{i \alpha} \\
& +\frac{U}{2} \sum_{i \alpha} c_{i \alpha}^{\dagger} c_{i \alpha}\left(c_{i \alpha}^{\dagger} c_{i \alpha}-1\right) .
\end{aligned}
$$

Here, $c_{i \alpha}$ is a bosonic annihilation operator for the $\alpha$ th sublattice site within the $i$ th unit cell; the matrix $\mathcal{H}$ contains the hopping coefficients between different sites; $\mu$ is the 


\section{DISPERSIVE BAND}

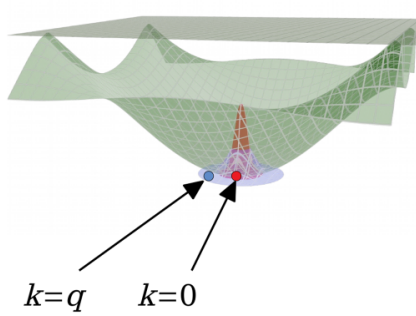

$n_{0}$ Condensate density $n_{e}$ Excitation density $U$ Interaction $u(k)$ Bloch function

\section{SPEED OF SOUND}

$$
\begin{array}{cc}
C_{s} \propto \sqrt{U n_{0} / m_{\text {eff }}} & U n_{0} \sqrt{g_{\alpha \beta}\left(k_{c}\right)} \\
& \text { Quantum metric } \\
g_{\alpha \beta}=\Re\left[\left\langle\partial_{\alpha} u \mid \partial_{\beta} u\right\rangle-\left\langle\partial_{\alpha} u \mid u\right\rangle\left\langle u \mid \partial_{\beta} u\right\rangle\right]
\end{array}
$$

\section{EXCITATION DENSITY (Quantum depletion)}

$$
n_{e}(q) \underset{U \rightarrow 0}{\longrightarrow} 0
$$

$$
n_{e}(k) \underset{U \rightarrow 0}{\longrightarrow} \frac{1-D}{D}
$$

Quantum distance

$$
D=\sqrt{1-\left|\left\langle u\left(k_{c}+q\right) \mid u\left(k_{c}-q\right)\right\rangle\right|}
$$

\section{DENSITY-DENSITY CORRELATIONS}

$$
\begin{aligned}
& \langle n n\rangle \sim\left\langle n_{0} n_{0}\right\rangle+\left\langle n_{e} n_{0}\right\rangle+\underbrace{\left\langle n_{e} n_{e}\right\rangle}_{\text {Negligible }}\langle n n\rangle \sim\left\langle n_{0} n_{0}\right\rangle+\left\langle n_{e} n_{0}\right\rangle+\underbrace{\left\langle n_{e} n_{e}\right\rangle}_{\text {Can }} \\
& \text { SUPERFLUID WEIGHT } \\
& \text { dominate }
\end{aligned}
$$

$$
D_{s} \sim \frac{n_{0}}{m^{*}} \quad D_{s} \text { determined by fluctuations }
$$

FIG. 1. The connection between flat-band Bose-Einstein condensation and quantum geometry. The right panel summarizes the main results of this paper; for comparison, the left panel shows known results on dispersive band BEC. Here we show results for the case of a kagome flat-band model, however, the general formulas (see text) have essentially similar dependence on the geometric quantities.

chemical potential; $U>0$ is the repulsive onsite interaction. The sublattice index ranges from 1 to $M$, where $M$ is the number of lattice sites per unit cell. By assuming periodic boundary conditions and introducing the Fourier transforms $c_{i \alpha}=\frac{1}{\sqrt{N}} \sum_{\mathbf{k}} \exp \left(i \mathbf{k} \cdot \mathbf{r}_{i \alpha}\right) c_{\mathbf{k} \alpha}$, where $N$ is the number of unit cells, $\mathbf{r}_{i \alpha}$ is the location of the $\alpha$ th lattice site in the $i$ th unit cell, and $\mathbf{k}$ is the momentum, one gets

$$
\begin{aligned}
H= & \sum_{\mathbf{k}}\left(c_{\mathbf{k}}^{\dagger} \mathcal{H}(\mathbf{k}) c_{\mathbf{k}}-\mu c_{\mathbf{k}}^{\dagger} c_{\mathbf{k}}\right) \\
& +\frac{U}{2 N} \sum_{\alpha} \sum_{\mathbf{k}, \mathbf{k}^{\prime}, \mathbf{q}} c_{\mathbf{k} \alpha}^{\dagger} c_{\mathbf{k}-\mathbf{q} \alpha} c_{\mathbf{k}^{\prime} \alpha}^{\dagger} c_{\mathbf{k}^{\prime}+\mathbf{q} \alpha} .
\end{aligned}
$$

Here, the one-particle Hamiltonian $\mathcal{H}(\mathbf{k})$ is a $M \times M$ matrix and $c_{\mathbf{k}}$ is a $M \times 1$ vector such that $\left[c_{\mathbf{k}}\right]_{\alpha}=c_{\mathbf{k} \alpha}$. One can diagonalize $\mathcal{H}(\mathbf{k})$ as $\mathcal{H}(\mathbf{k})\left|u_{n}(\mathbf{k})\right\rangle=\epsilon_{n}(\mathbf{k})\left|u_{n}(\mathbf{k})\right\rangle$, where $\epsilon_{n}(\mathbf{k})$ $\left(\left|u_{n \mathbf{k}}\right\rangle\right)$ are the eigenenergies (Bloch states) and $n$ is the band index so that $\epsilon_{1}(\mathbf{k}) \leqslant \epsilon_{2}(\mathbf{k}) \leqslant \cdots \leqslant \epsilon_{M}(\mathbf{k})$.

As we consider an equilibrium situation, the condensation takes place within the lowest Bloch band. Furthermore, we are mainly interested in condensation occurring within a flat or quasiflat Bloch band; the latter is defined by $J \ll U n_{0}$, where $J$ is the width of the band and $n_{0}$ is the number of condensed bosons per unit cell. Since the Bloch states of the flat band are degenerate in energy, the following question arises: On which state does the condensation occur? To answer this, we use the approach of Ref. [62], i.e., utilize the mean-field (MF) approximation [65], where we substitute operators in Eq. (2) by complex numbers, and the resulting MF energy $E_{\mathrm{MF}}(\mathbf{k})$ is solved separately for each $\mathbf{k}$ with fixed density $n_{0}$ (see Appendix A). The condensation takes place at the Bloch state $\left|\phi_{0}\right\rangle \equiv\left|u_{1}\left(\mathbf{k}_{c}\right)\right\rangle$ that minimizes $E_{\mathrm{MF}}(\mathbf{k})$ [62]. The momentum (energy) of the condensed Bloch state is denoted as $\mathbf{k}_{c}\left[\epsilon_{0} \equiv \epsilon_{1}\left(\mathbf{k}_{c}\right)\right]$.

Therefore, even if the lowest Bloch band is strictly flat, the condensate can still occur at some specific Bloch state, as the repulsive onsite interaction favors Bloch states that distribute the particles among the sublattices as equally as possible. We herein assume uniform condensate density, i.e., $\left|\left\langle\alpha \mid \phi_{0}\right\rangle\right|^{2}=$ $1 / M$ for all $\alpha$ with $\left\langle\alpha \mid \phi_{0}\right\rangle$ being the projection of $\left|\phi_{0}\right\rangle$ to the orbital $\alpha$. This is a rather general condition; examples will be presented below.

To analyze the excitations of the condensate, we express $c_{i \alpha}$ as

$$
c_{i \alpha}=\sqrt{n_{0}} \psi_{0}\left(\mathbf{r}_{i \alpha}\right)+\delta c_{i \alpha} \equiv c_{\alpha}^{0}+\delta c_{i \alpha} .
$$

Here, $\psi_{0}\left(\mathbf{r}_{i \alpha}\right)=\exp \left(i \mathbf{k}_{c} \cdot \mathbf{r}_{i \alpha}\right)\left\langle\alpha \mid \phi_{0}\right\rangle$ is the wave function of the condensate with the wave vector $\mathbf{k}_{c}$ and $\delta c_{i \alpha}$ describes the fluctuations on top of the condensate. By Fourier transforming, one finds $c_{\mathbf{k}_{c} \alpha}=\sqrt{N n_{0}}\left\langle\alpha \mid \phi_{0}\right\rangle$ and $c_{\mathbf{k} \neq \mathbf{k}_{c} \alpha}=$ $N^{-1 / 2} \sum_{i} e^{-i \mathbf{k} \cdot \mathbf{r}_{i \alpha}} \delta c_{i \alpha}$.

We now treat the Hamiltonian within the multiband Bogoliubov approximation (for details, see Appendix B) by neglecting the interaction terms that are higher than quadratic order in the fluctuations $c_{\mathbf{k} \alpha}$ and $c_{\mathbf{k} \alpha}^{\dagger}$ with $\mathbf{k} \neq \mathbf{k}_{c}$. One can then express Eq. (1) as $H=E_{c}+H_{B}$, where $E_{c}$ is a constant giving the ground energy of the condensate. The excitations are described by the Bogoliubov Hamiltonian (see Appendix B for details)

$$
H_{B}=\frac{1}{2} \sum_{\mathbf{k}}{ }^{\prime} \Psi_{\mathbf{k}}^{\dagger} \mathcal{H}_{B}(\mathbf{k}) \Psi_{\mathbf{k}},
$$

where $\mathcal{H}_{B}(\mathbf{k})$ is a $2 M \times 2 M$ matrix given by

$$
\begin{aligned}
\mathcal{H}_{B}(\mathbf{k}) & =\left[\begin{array}{cc}
\mathcal{H}(\mathbf{k})-\mu_{\mathrm{eff}} & \Delta \\
\Delta^{*} & \mathcal{H}^{*}\left(2 \mathbf{k}_{c}-\mathbf{k}\right)-\mu_{\mathrm{eff}},
\end{array}\right], \\
\Psi_{\mathbf{k}} & =\left[c_{\mathbf{k} 1}, c_{\mathbf{k} 2}, \ldots, c_{\mathbf{k} M}, c_{2 \mathbf{k}_{c}-\mathbf{k} 1}^{\dagger}, \ldots, c_{2 \mathbf{k}_{c}-\mathbf{k} M}^{\dagger}\right]^{T}, \\
{[\Delta]_{\alpha \beta} } & =\delta_{\alpha, \beta} U n_{0}\left\langle\alpha \mid \phi_{0}\right\rangle^{2}, \\
\mu_{\mathrm{eff}} & =\left(\epsilon_{0}-\frac{U n_{0}}{M}\right) \delta_{\alpha, \beta} .
\end{aligned}
$$

The primed sum in Eq. (4) indicates that all the operators within the sum are for noncondensed states only, i.e., $\mathbf{k} \neq \mathbf{k}_{c}$ and $2 \mathbf{k}_{c}-\mathbf{k} \neq \mathbf{k}_{c}$.

To obtain the excitation energies of the condensate, one cannot directly diagonalize $\mathcal{H}_{B}$ since this would violate the bosonic commutation relations. Instead, one needs to find the eigenstates of $L(\mathbf{k}) \equiv \sigma_{z} \mathcal{H}_{B}(\mathbf{k})$, where $\sigma_{z}$ is the Pauli matrix [66]. One obtains Bogoliubov bands of the energies $E_{M}(\mathbf{k}) \geqslant \cdots E_{2}(\mathbf{k}) \geqslant E_{1}(\mathbf{k}) \geqslant 0 \geqslant-E_{1}\left(2 \mathbf{k}_{c}-\right.$ $\mathbf{k}) \geqslant \cdots-E_{M}\left(2 \mathbf{k}_{c}-\mathbf{k}\right)$. Here, positive (negative) energies describe quasiparticle (quasihole) excitations. The quasipar- 
ticle and quasihole states are labeled as $\left|\psi_{m}^{+}(\mathbf{k})\right\rangle$ and $\left|\psi_{m}^{-}(\mathbf{k})\right\rangle$ such that

$$
\begin{aligned}
L(\mathbf{k})\left|\psi_{m}^{+}(\mathbf{k})\right\rangle & =E_{m}(\mathbf{k})\left|\psi_{m}^{+}(\mathbf{k})\right\rangle, \\
L(\mathbf{k})\left|\psi_{m}^{-}(\mathbf{k})\right\rangle & =-E_{m}\left(2 \mathbf{k}_{c}-\mathbf{k}\right)\left|\psi_{m}^{-}(\mathbf{k})\right\rangle .
\end{aligned}
$$

The chemical potential in Eq. (4) is fixed such that the lowest quasiparticle energy band is gapless at $\mathbf{k}_{c}$, i.e., $E_{1}\left(\mathbf{k} \rightarrow \mathbf{k}_{c}\right)=$ 0 . This is the usual Goldstone mode that emerges because the condensate wave function spontaneously picks up a complex phase, leading to the spontaneous gauge $\mathrm{U}(1)$ symmetry breaking $[63,65]$.

\section{SPEED OF SOUND AT THE WEAK-COUPLING REGIME}

After setting up the Bogoliubov theory, we now proceed to derive our main results. We first compute the speed of sound $c_{s}$ for small $U$, which is given by the slope of the lowest Bogoliubov excitation band in the long-wavelength limit:

$$
c_{s}\left(\theta_{\mathbf{q}}\right)=\lim _{|\mathbf{q}| \rightarrow 0} \frac{E_{1}\left(\mathbf{k}_{c}+\mathbf{q}\right)-E_{1}\left(\mathbf{k}_{c}\right)}{|\mathbf{q}|}=\lim _{|\mathbf{q}| \rightarrow 0} \frac{E_{1}\left(\mathbf{k}_{c}+\mathbf{q}\right)}{|\mathbf{q}|},
$$

with $|\mathbf{q}| \ll 1$ (we use units where the lattice constant is unity). In general, $c_{s}$ depends on the direction of its momentum $\mathbf{q}$, parametrized by the angle $\theta_{\mathbf{q}}$ between $\mathbf{q}$ and the $x$ axis. We assume that around $\mathbf{k}_{c}$, the lowest Bloch band is isolated from the higher Bloch bands by an energy gap $E_{g}$ that is large compared to the interaction energy, i.e., $U n_{0} \ll E_{g}$. In this case, the interaction term does not cause notable mixing between the lowest and dispersive bands. This is important as we are interested in the properties of flat-band BECs. In the large interaction regime with $U n_{0} \gg E_{g}$, the importance of a flat band would be obscured by the dispersive bands. With $U n_{0} \ll E_{g}$, we therefore discard the higher bands and project to the lowest band to obtain (see Appendix C)

$$
L_{p}(\mathbf{k})=\left[\begin{array}{cc}
\frac{\mathbf{q}^{2}}{2 m_{e f f}}+\frac{U n_{0}}{M} & \frac{U n_{0}}{M} \alpha(\mathbf{q}) \\
-\frac{U n_{0}}{M} \alpha^{*}(\mathbf{q}) & -\frac{\mathbf{q}^{2}}{2 m_{\text {eff }}}-\frac{U n_{0}}{M}
\end{array}\right]
$$

for $\mathbf{q} \rightarrow 0$. Here, the subscript $p$ indicates that this is the projected $2 \times 2$ matrix. We have furthermore defined the off-diagonal term as $\alpha(\mathbf{q}) \equiv \frac{M}{U n_{0}}\left\langle u_{1}\left(\mathbf{k}_{c}+\mathbf{q}\right)|\Delta| u_{1}^{*}\left(\mathbf{k}_{c}-\mathbf{q}\right)\right\rangle$, which contains information about the quantum geometric properties of the Bloch states. It is this quantity that encodes quantum geometric effects to various physical properties of a BEC taking place in a multiband system, as can be strikingly seen in the flat-band limit shown below. To contrast geometric effects to the behavior of a conventional BEC, we have retained a possible finite dispersion relation of the lowest band via the term $\mathbf{q}^{2} / 2 m_{\text {eff. }}$.

It is easy to obtain $E_{1}\left(\mathbf{k}_{c}+\mathbf{q}\right)$ by diagonalizing Eq. (9), which yields

$$
E_{1}\left(\mathbf{k}_{c}+\mathbf{q}\right)=\sqrt{\left(\frac{U n_{0}}{M}\right) \frac{\mathbf{q}^{2}}{m_{\mathrm{eff}}}+\left(\frac{U n_{0}}{M}\right)^{2}\left[1-|\alpha(\mathbf{q})|^{2}\right]}
$$

for small $|\mathbf{q}|$. The two terms inside the square root in Eq. (10) have a completely different origin. The first is the usual term arising from the dispersion of the bosons in the considered Bloch band, which vanishes for a strictly flat band, i.e., when $m_{\text {eff }} \rightarrow \infty$. On the other hand, the second term involves overlaps of the Bloch states, and its connection to quantum geometry has not been considered in the context of Bose-Einstein condensation before. The quantity $\alpha(\mathbf{q})$ reads as

$$
\alpha(\mathbf{q})=M \sum_{\alpha}\left\langle u_{1}\left(\mathbf{k}_{c}+\mathbf{q}\right) \mid \alpha\right\rangle\left\langle\alpha \mid \phi_{0}\right\rangle\left\langle\phi_{0}^{*} \mid \alpha\right\rangle\left\langle\alpha \mid u_{1}^{*}\left(\mathbf{k}_{c}-\mathbf{q}\right)\right\rangle,
$$

which gives an overlap between $\left|\phi_{0}\right\rangle$ and the states of the particle $\left|u_{1}\left(\mathbf{k}_{c}+\mathbf{q}\right)\right\rangle$ and the hole $\left|u_{1}^{*}\left(\mathbf{k}_{c}-\mathbf{q}\right)\right\rangle$.

Defining $\tilde{D}(\mathbf{q}) \equiv \sqrt{1-|\alpha(\mathbf{q})|^{2}}$, we can write Eq. (10) as

$$
E_{1}\left(\mathbf{k}_{c}+\mathbf{q}\right)=\frac{U n_{0}}{M} \tilde{D}(\mathbf{q})
$$

for a flat band with $m_{\mathrm{eff}} \rightarrow \infty$. This shows that the Bogoliubov excitation energies for a flat band are determined by $\tilde{D}(\mathbf{q})$, which we call the "condensate quantum distance" between the condensed state and the neighboring states, since $\tilde{D}(\mathbf{q})=0$ when $\left\langle u_{1}\left(\mathbf{k}_{c}+\mathbf{q}\right) \mid \phi_{0}\right\rangle=\left\langle u_{1}\left(\mathbf{k}_{c}-\mathbf{q}\right) \mid \phi_{0}\right\rangle=1$. Indeed, $\tilde{D}(\mathbf{q})$ becomes identical to the Hilbert-Schmidt quantum distance $D(\mathbf{q})[15,67]$

$$
D(\mathbf{q}) \equiv \sqrt{1-\left|\left\langle u_{1}\left(\mathbf{k}_{c}+\mathbf{q}\right) \mid u_{1}\left(\mathbf{k}_{c}-\mathbf{q}\right)\right\rangle\right|^{2}}
$$

when $\left|u_{1}^{*}(\mathbf{k})\right\rangle=\left|u_{1}(\mathbf{k})\right\rangle$. By Taylor expanding up to second order in $\mathbf{q}$, one has $D^{2}(\mathbf{q})=4 \sum_{\mu \nu} q_{\mu} q_{\nu} g_{\mu \nu}^{1}\left(\mathbf{k}_{c}\right)$, where

$$
g_{\mu \nu}^{n}(\mathbf{k})=\operatorname{Re}\left[\left\langle\partial_{\mu} u_{n}(\mathbf{k})\left|\left(1-\left|u_{n}(\mathbf{k})\right\rangle\left\langle u_{n}(\mathbf{k})\right|\right)\right| \partial_{\nu} u_{n}(\mathbf{k})\right\rangle\right]
$$

is the quantum metric $g_{\mu \nu}^{n}(\mathbf{k})$ of the $n$th Bloch band and $\partial_{\mu} \equiv$ $\frac{\partial}{\partial q_{\mu}}$. This implies that the speed of sound for the flat band, $c_{s, f . b .}$, reads as

$$
c_{s, f . b .}\left(\theta_{\mathbf{q}}\right)=\frac{2 U n_{0}}{M} \sqrt{\hat{\mathbf{e}}_{\mathbf{q}}^{T} g^{1}\left(\mathbf{k}_{c}\right) \hat{\mathbf{e}}_{\mathbf{q}}}, \text { with }\left|u_{1}^{*}(\mathbf{k})\right\rangle=\left|u_{1}(\mathbf{k})\right\rangle
$$

is purely determined by the quantum metric. Here, $\hat{\mathbf{e}}_{\mathbf{q}}=$ $\mathbf{q} /|\mathbf{q}|, \tan \theta_{\mathbf{q}}=q_{y} / q_{x}$, and $\left[g^{1}\right]_{\mu \nu}=g_{\mu \nu}^{1}$.

From Eq. (15) we see that finite quantum metric yields nonzero $c_{s}$ and thus allows superfluidity even in case of a flat-band BEC. This result can be contrasted to fermionic flat-band systems where superconductivity was shown to exist for nonzero Chern numbers or momentum-space integrals of of the quantum metric [10-12]. The difference to the present bosonic BEC case is that only the quantum metric of the condensed Bloch state $\left|\phi_{0}\right\rangle$ matters, not the geometric quantities integrated over the whole BZ. Furthermore, the speed of sound of a spin-orbit coupled Fermi gas was studied in Ref. [68]: $c_{s}$ was shown to partially depend on the quantum geometric terms but their significance was hampered due to the presence of more dominant nongeometric contributions. In our case, quantum geometry manifests as the sole contribution for the weakly coupled flat-band BEC.

In the general case of $\left|u_{1}^{*}(\mathbf{k})\right\rangle \neq\left|u_{1}(\mathbf{k})\right\rangle$, we use the condition $\left|\left\langle\alpha \mid \phi_{0}\right\rangle\right|^{2}=1 / M$ and expand $\left|u_{1}\left(\mathbf{k}_{c}+\mathbf{q}\right)\right\rangle$ and $\mid u_{1}^{*}\left(\mathbf{k}_{c}-\right.$ 


\section{Kagome:}

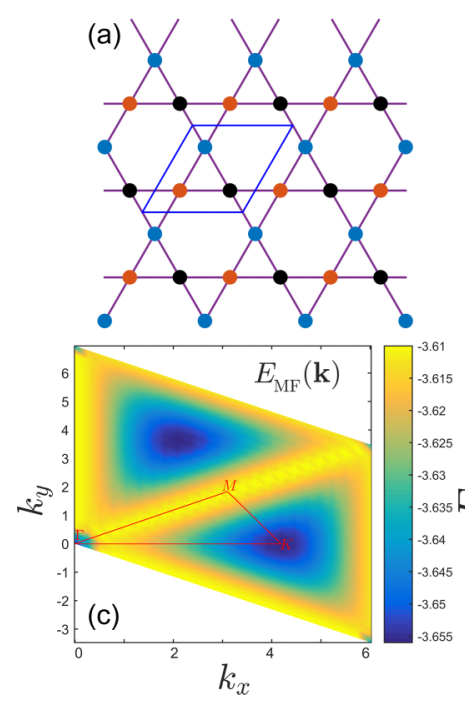

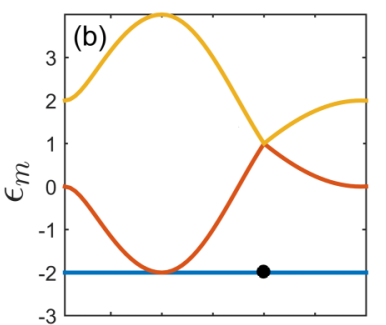

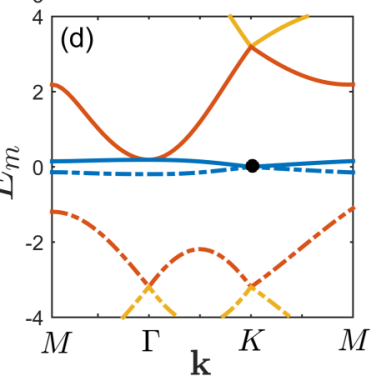

CB-III:
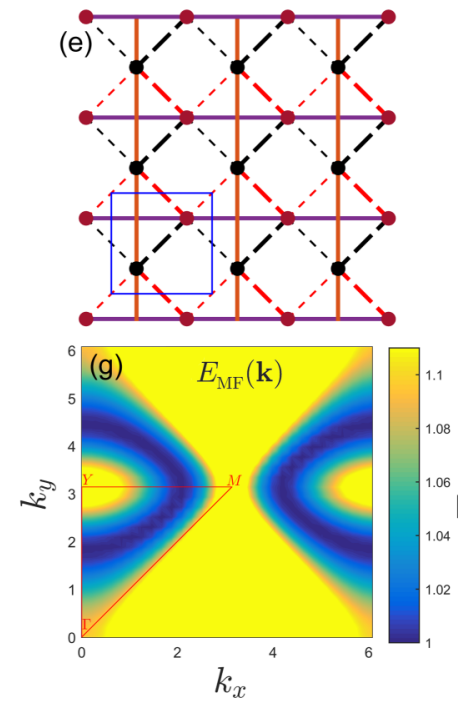
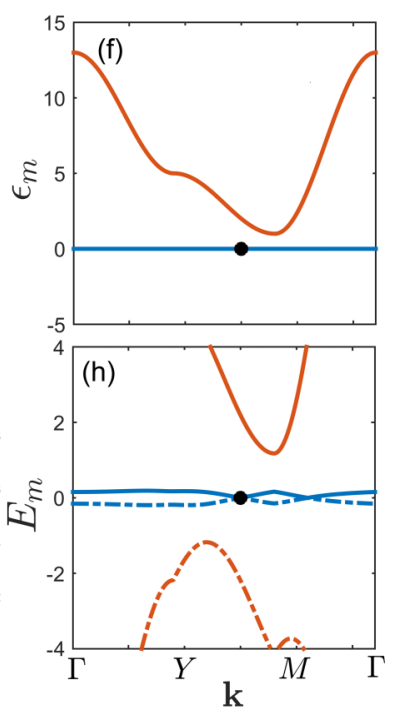

FIG. 2. Flat-band models considered in this work. (a) Kagome lattice geometry. The unit cell is shown as a blue parallelogram. Purple lines depict NN hopping terms of strength $t$. (b) Bloch bands of the kagome lattice with $t=1$ along high-symmetry points. (c) Mean-field energy $E_{\mathrm{MF}}(\mathbf{k})$ across the BZ for the kagome lattice. It is minimized at the $\Gamma$ and Dirac points but fluctuation analysis [62] shows that the Dirac-point condensate is the most favorable. The red lines depict the path along which the dispersions in (b) and (d) are plotted. (d) Bogoliubov spectrum for the kagome lattice along high-symmetry lines with $\mathbf{k}_{c}=[4 \pi / 3,0]$ [marked as a black dot in (b) and (d)] and $U n_{0} / M t=0.2$. Quasiparticle (quasihole) modes are depicted with solid (dashed) lines. There is a gapless Goldstone mode at $\mathbf{k}_{c}$. (e) Checkerboard-III (CB-III) geometry. The blue square represents the unit cell. Solid purple (orange) lines depict kinetic hopping terms of strength $t=1$ ( $2 t$ ), whereas bolded black (red) dashed lines are hoppings of strength $-2 t(-t)$. Thin black and red dashed lines depict hoppings of strength $-2 t(1-\delta)$ and $-t(1-\delta)$, where a finite staggering parameter $\delta \ll 1$ can be used to render the flat Bloch band slightly dispersive. In the computations we use $\delta=10^{-5}$. The sublattice depicted as black (red) dots has the onsite energy of $5 t(2 t)$. (f) Bloch band structure for CB-III. (g) Mean-field energy $E_{\mathbf{M F}}(\mathbf{k})$ for the CB-III model with $\delta=0$. The red lines depict the path along which the dispersions in (f) and (h) are plotted. There is a continuous subset of Bloch states that minimize $E_{\mathbf{k}}$ equally well. By using a finite $\delta$, one can introduce stable condensation to the momentum $\mathbf{k}=[2 \pi / 3, \pi]$. (h) Bogoliubov spectrum for the CB-III model along high-symmetry lines with $\mathbf{k}_{c}=[2 \pi / \pi, \pi]$ [marked as a black dot in (b) and (d)] and $\delta=10^{-5}$ at $U n_{0} / M t=0.2$. Quasiparticle (quasihole) modes are depicted with solid (dashed) lines. A gapless Goldstone mode exists at $\mathbf{k}_{c}$. A small gap opens at the $M$ point, despite being barely visible in the plot.

q) $\rangle$ up to second order in q. This gives

$$
\begin{aligned}
\tilde{D}^{2}(\mathbf{q})= & \sum_{\mu \nu} q_{\mu} q_{\nu}\left\{2 g_{\mu \nu}^{1}\left(\mathbf{k}_{c}\right)+2 \operatorname{Re}\left[\left\langle\partial_{\mu} \phi_{0} \mid \phi_{0}\right\rangle\left\langle\phi_{0} \mid \partial_{\nu} \phi_{0}\right\rangle\right]\right. \\
& \left.+2 M \operatorname{Re}\left[\sum_{\alpha}\left\langle\partial_{\mu} \phi_{0} \mid \alpha\right\rangle\left\langle\alpha \mid \phi_{0}\right\rangle\left\langle\phi_{0}^{*} \mid \alpha\right\rangle\left\langle\alpha \mid \partial_{\nu} \phi_{0}^{*}\right\rangle\right]\right\} \\
\equiv & 4 \sum_{\mu \nu} q_{\mu} q_{\nu} \tilde{g}_{\mu \nu}\left(\mathbf{k}_{c}\right)
\end{aligned}
$$

for $\mathbf{q} \rightarrow 0$. We have introduced a generalized metric $\tilde{g}_{\mu \nu}$, which replaces $g_{\mu \nu}^{1}$ in Eq. (15) for the speed of sound when $\left|u_{1}^{*}(\mathbf{k})\right\rangle \neq\left|u_{1}(\mathbf{k})\right\rangle$. Consequently, the discussion following Eq. (15) remains valid for a general flat band with the replacement $g_{\mu \nu} \rightarrow \tilde{g}_{\mu \nu}$. With Eq. (16), Eq. (10) and $c_{s, f . b .}$ can be recast as

$$
\begin{aligned}
E_{1}\left(\mathbf{k}_{c}+\mathbf{q}\right) & =\sqrt{\left(\frac{U n_{0}}{M m_{\mathrm{eff}}}\right) \mathbf{q}^{2}+\left(\frac{2 U n_{0}}{M}\right)^{2} \sum_{\mu \nu} q_{\mu} q_{\nu} \tilde{g}_{\mu \nu}\left(\mathbf{k}_{c}\right),} \\
c_{c, f . b .} & =\frac{2 U n_{0}}{M} \sqrt{\hat{\mathbf{e}}_{\mathbf{q}}^{T} \tilde{g}\left(\mathbf{k}_{c}\right) \hat{\mathbf{e}}_{\mathbf{q}}} .
\end{aligned}
$$

When the geometric contribution is zero, i.e., $\tilde{g}_{\mu \nu}=0$, the speed of sound for a dispersive band reduces to the usual form $c_{s}=\sqrt{U n_{0} /\left(M m_{\text {eff }}\right)}$, i.e., $c_{s} \propto \sqrt{U}$ [65]. This should be compared to the flat-band limit $m_{\text {eff }} \rightarrow \infty$, where the first term in Eq. (17) vanishes and $c_{s} \propto U$. The linear vs square-root dependence can be used to distinguish the sound velocities of geometric and conventional origin in an experiment where $U$ can be tuned. Equivalently, one can define an interaction-dependent effective mass for the flat band $\tilde{m}_{\text {eff }}=$ $M /\left(4 U n_{0} \hat{\mathbf{e}}_{\mathbf{q}}^{T} \tilde{g} \hat{\mathbf{e}}_{\mathbf{q}}\right)$ so that the speed of sound is formally the same as for a dispersive band $c_{s}=\sqrt{U n_{0} /\left(M \tilde{m}_{\mathrm{eff}}\right)}$. Different forms of $c_{s}$ for dispersive and flat-band condensates are summarized in Fig. 1.

\section{Speed of sound in kagome and checkerboard lattices}

We now consider $c_{s}$ in two specific flat-band models. The kagome lattice [see Fig. 2(a)] consists of three sublattices, and one of the three Bloch bands is strictly flat [Fig. 2(b)] [62]. When the nearest-neighbor (NN) hopping is positive $t>0$, the flat band has the lowest energy and Bose condensation can take place within it. By minimizing the mean-field energy $E_{\mathrm{MF}}(\mathbf{k})$, one finds [62] that condensation at the $\Gamma$ point, i.e., $\mathbf{k}=0$, or at one of the Dirac points, e.g., $\mathbf{k}_{c}=\mathbf{k}_{K} \equiv$ $[4 \pi / 3,0]$, is favored [see Fig. 2(c)] since the particle density 

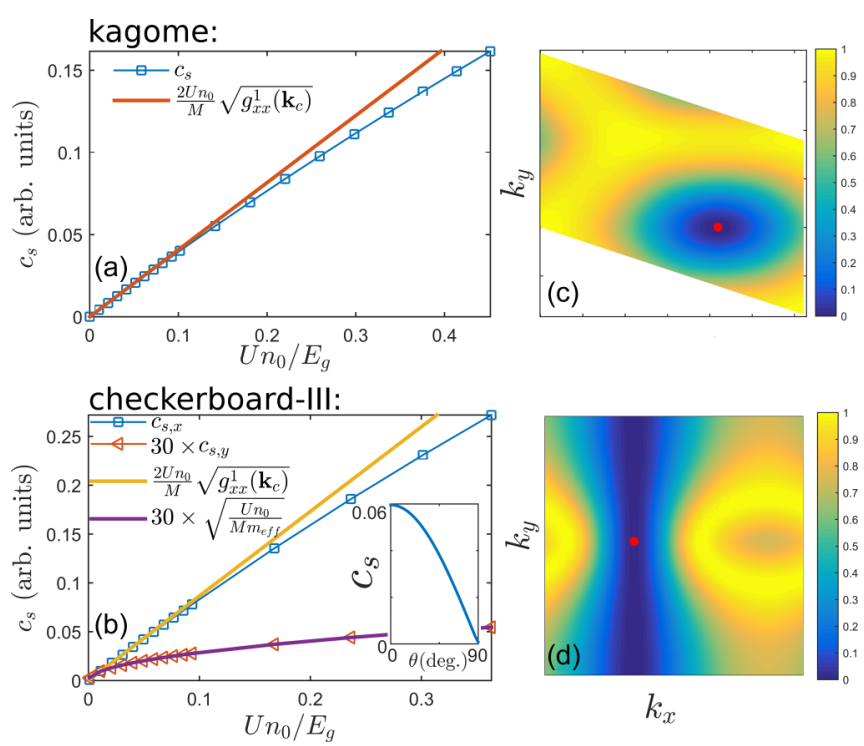

FIG. 3. Speed of sound in flat-band condensates. (a), (b) The speed of sound $c_{s}$ for kagome and CB-III flat-band condensates, respectively, as a function of $U$. Total density was chosen to be $n_{\text {tot }}=M$, i.e., one particle per lattice site. We also show the weakcoupling result of Eq. (15). The quasi-flat band of CB-III has a small but nonzero bandwidth $J \sim 10^{-5} U n_{0}$ due to a finite $\delta$. For the kagome model, $c_{s}$ is determined by the quantum metric of the condensed state, which in this case is isotropic. In CB-III, $c_{s}$ in the $x$ direction $\left(c_{s, x}\right)$ depends on the quantum metric and in the $y$ direction $\left(c_{s, y}\right)$ on the effective mass $m_{\mathrm{eff}}$ of the quasi-flat band. The energy scaling $E_{g}$ is taken to be the energy gap between the flat band and higher bands at $\mathbf{k}_{c}$. To make it more visible, data for $c_{s, y}$ are scaled by a factor of 30 . Inset of (b) shows the angle dependence of $c_{s}$ for CB-III at $U n_{0} / E_{g}=0.07$. (c), (d) The condensate quantum distance $\tilde{D}(\mathbf{q})$ for the kagome and CB-III lattices as a function of $\mathbf{k}=\mathbf{k}_{c}+\mathbf{q}$. For the kagome (CB-III) lattice, $\tilde{D}$ is computed with respect to $\mathbf{k}_{c}=[4 \pi / 3,0]\left(\mathbf{k}_{c}=[2 \pi / 3, \pi]\right)$, marked as a red dot. For CB-III, $\tilde{D}(\mathbf{q})=0$ in the $k_{y}$ direction, consistent with the fact that for $\delta=0$ the condensation at $\mathbf{k}_{c}=[2 \pi / 3, \pi]$ is unstable.

distributes uniformly among the sublattices for these Bloch states, minimizing the repulsive onsite interaction. A fluctuation analysis [62] shows the condensation at $\mathbf{k}_{K}$ having a slightly smaller zero-point energy, being then more favorable than the $\Gamma$ condensate. We thus take $\mathbf{k}_{c}=\mathbf{k}_{K}$. Since the upper two dispersive bands are far away from the flat band at $\mathbf{k}_{K}$, the $\mathbf{k}_{K}$ condensate realizes flat-band condensation to a good approximation.

In Fig. 2(d) we show a typical Bogoliubov spectrum for the kagome flat-band condensate at $\mathbf{k}_{c}=\mathbf{k}_{K}$. A gapless Goldstone mode exists whose dispersion around $\mathbf{k}_{c}$ is linear. By extracting the slope of the Goldstone mode, we obtain $c_{s}$ which is plotted in Fig. 3(a) as a function of interaction $U$. Furthermore, because $\left|u_{1}^{*}\left(\mathbf{k}_{c}-\mathbf{q}\right)\right\rangle=\left|u_{1}\left(\mathbf{k}_{c}-\mathbf{q}\right)\right\rangle$, Eq. (15) holds for $U \rightarrow 0$. Thus, alongside the numerical result, we also plot in Fig. 3(a) the weak-coupling result of Eq. (15). The agreement for small interactions is excellent. Moreover, $c_{s}$ is isotropic, consistent with the fact that $\tilde{D}(\mathbf{q})$ around $\mathbf{k}_{c}$ is rotationally symmetric [see Fig. 3(c)].
As another example, we consider the checkerboard-III (CB-III) geometry [69] [Fig. 2(e)] that consists of two sublattices and features a strictly flat band [see Fig. 2(f)]. By minimizing the MF energy $E_{\mathrm{MF}}(\mathbf{k})$, one finds that there exists a continuous subset of flat-band Bloch states that minimize the condensation energy [Fig. 2(g)]. One of these states is at $\mathbf{k}=$ $[2 \pi / 3, \pi]$, and computing $\tilde{D}(\mathbf{q})$ for this state gives $\tilde{D}(\mathbf{q})=0$ at $\mathbf{q} \| \mathbf{e}_{y}$ as shown in Fig. 3(d). This means that $c_{s}=0$ in the $y$ direction, implying an unstable BEC. This is consistent with $E_{\mathrm{MF}}(\mathbf{k})$ being minimized for a continuous subset of flat-band states [see Fig. 2(g)], which makes condensation in a single Bloch state ambiguous.

Our results demonstrate an important aspect of flat-band condensation. While finite interactions are needed, they are not sufficient to obtain a stable flat-band BEC. An additional condition is to have a suitable quantum geometry for the flat band. Indeed, in the CB-III geometry, a flat-band BEC cannot be obtained as the quantum distance is zero between some of the flat-band states. Another example of an unstable flat-band BEC is a single-band model for which $\tilde{D}(\mathbf{q})=0$ for all $\mathbf{q}$. In case of the kagome lattice, we have shown above that the flat-band condensate can be stable due to a nonzero quantum distance between the Bloch states. The connection between the stability of a flat-band BEC and the quantum distance is elaborated further in the next section.

The checkerboard-III lattice condensation can be made stable by introducing a small perturbation to the $\mathrm{NN}$ hopping terms [Fig. 2(e)] that renders the lowest Bloch band slightly dispersive. By computing $c_{s}$ with Eq. (17), one finds that $c_{s}$ in the $y$ direction scales as $\sqrt{U}$ due to finite dispersion. In the $x$ direction, however, $c_{s}$ scales linearly in $U$ and is therefore determined by the geometric term $\tilde{D}(\mathbf{q})$. Although the condition $\left|u_{1}^{*}\right\rangle=\left|u_{1}\right\rangle$ is not strictly met, $c_{s}$ in the $x$ direction is still mostly dictated by the quantum metric [see Fig. 3(b)]. By computing $c_{s}$ for all the directions $\theta_{\mathbf{q}}$, we see that $c_{s}$ decreases monotonically between the directions $\theta_{\mathbf{q}}=0$ and $\theta_{\mathbf{q}}=\pi / 2$ [see inset of Fig. 3(b)], reflecting the anisotropy of $\tilde{D}(\mathbf{q})$ shown in Fig. 3(d).

\section{EXCITATION FRACTION}

In addition to the speed of sound, quantum geometry can manifest also via other physical quantities. We demonstrate this here for the excitation density $n_{\mathrm{ex}}$, i.e., the number of noncondensed bosons per unit cell, in the limit of $U \rightarrow 0$. In case of the usual dispersive band condensate, one has $\lim _{U \rightarrow 0} n_{\text {ex }}=0$ at zero temperature $[63,65]$. However, for a strictly flat band, the limit $U \rightarrow 0$ implies that the lowest Bogoliubov excitation band becomes flat as the off-diagonal terms of $\mathcal{H}_{B}$ in Eq. (5) vanish, i.e., $\Delta \rightarrow 0$. This implies that the BEC at a single-flat-band state becomes unstable (see also Appendix D) as the lowest Bogoliubov band excitations have vanishing energy cost. Therefore, it is not intuitively clear what kind of asymptotic behavior $\lim _{U \rightarrow 0} n_{\mathrm{ex}}$ of a flat-band condensate features. In this section we show that, remarkably, $n_{\mathrm{ex}}$ can be nonzero and finite for vanishing interaction strength, and that its value is dictated by quantum geometry. 
The expression for $n_{\text {ex }}$ can be written as (see Appendix D for details)

$$
\begin{aligned}
n_{\mathrm{ex}} & =\frac{1}{N} \sum_{\mathbf{k} m}{ }^{\prime}\left\langle c_{\mathbf{k} m}^{\dagger} c_{\mathbf{k} m}\right\rangle=\frac{1}{2 N} \sum_{\mathbf{k} m}{ }^{\prime}\left[-1+\left\langle\psi_{m}^{-}(\mathbf{k}) \mid \psi_{m}^{-}(\mathbf{k})\right\rangle\right] \\
& =\frac{1}{N} \sum_{\mathbf{k}}{ }^{\prime} n_{\mathrm{ex}}(\mathbf{k})
\end{aligned}
$$

where $c_{\mathbf{k} m}^{\dagger}$ creates a boson in the Bloch band $m$ with momentum $\mathbf{k}$ and energy $\epsilon_{m}(\mathbf{k})$, obtained by diagonalizing $\mathcal{H}(\mathbf{k})$ in (5).

It is instructive to consider first a single-band case for which the one-particle energy spectrum reads as $\epsilon(\mathbf{k}) \propto$ $\mathbf{k}^{2} / m_{\text {eff }}$ corresponding to a continuum system or the longwavelength limit of a square lattice. The corresponding Bogoliubov energy is then $E_{1}(\mathbf{k})=\sqrt{\tilde{\epsilon}(\mathbf{k})\left[\tilde{\epsilon}(\mathbf{k})+2 U n_{0}\right]}$ with $\tilde{\epsilon}(\mathbf{k}) \equiv \epsilon(\mathbf{k})-\epsilon_{0}$, and one has $n_{\mathrm{ex}}(\mathbf{k})=[\tilde{\epsilon}(\mathbf{k})+$ $\left.U n_{0}\right] / E_{1}(\mathbf{k})-1$ [63]. We see that $n_{\text {ex }}$ diverges in the flat-band limit $1 / m_{\text {eff }}=0$. Thus, a flat-band BEC within a single-band system is always unstable. This is consistent with the results of the previous sections since for a single-band lattice system one always has $\alpha(\mathbf{k})=1$. Consequently, one needs to incorporate quantum geometric effects to reach a stable BEC, i.e., consider a multiband system.

We now focus on a multiband lattice system, assuming the condensation to take place within a strictly flat band and neglecting higher bands, which is justified for a small $U$. We therefore deploy Eq. (9) with $1 / m_{\text {eff }}=0$. Solving $L_{p}(\mathbf{k})\left|\psi_{1}^{-}(\mathbf{k})\right\rangle=-E_{1}(\mathbf{k})\left|\psi_{1}^{-}(\mathbf{k})\right\rangle$ and taking into account the proper normalization requirements [Eq. (B9) in Appendix B] gives

$$
\lim _{U \rightarrow 0}\left\langle c_{\mathbf{k} 1}^{\dagger} c_{\mathbf{k} 1}\right\rangle=\frac{1-\tilde{D}(\mathbf{q})}{2 \tilde{D}(\mathbf{q})},
$$

where $\mathbf{q}=\mathbf{k}-\mathbf{k}_{c}$. This expression relates a simple way the density of noncondensed bosons to the condensate quantum distance $\tilde{D}(\mathbf{q})$. It is valid for infinitesimally small but finite $U$; in complete absence of interactions $(U=0)$, a BEC in a strictly flat band cannot exist since all momentum states remain degenerate, as discussed in Sec. II.

The functional form of Eq. (20) is depicted in Fig. 4(a). If $\tilde{D}(\mathbf{q})=0, n_{\mathrm{ex}}(\mathbf{k})$ diverges, implying the breakdown of the Bogoliubov theory. This is intuitively easy to comprehend as a consequence of a perfect overlap between $\left|\phi_{0}\right\rangle$ and noncondensed Bloch states, which leads the particles to "spill" out of the condensate. It is also in agreement with the results presented in the previous section where $\tilde{D}(\mathbf{q})=0$ indicated unstable flat-band condensation.

To illustrate the role of the condensate quantum distance, we now consider $n_{\mathrm{ex}}$ in case of the flat-band BEC of the kagome lattice. For comparison, we also consider the dispersive-band $\mathrm{BEC}$ by carryring out the calculations for negative $\mathrm{NN}$ hopping term, i.e., $t=-1$. With this choice, one of two dispersive Bloch bands is the lowest one in which the bosons condense at $\mathbf{k}_{c}=0$.

In Fig. 4(b) we plot $n_{\mathrm{ex}} / n_{\text {tot }}$ (with $n_{\text {tot }}$ being the total density) as a function of interaction for the flat- and dispersive-band condensates. In the case of the dispersiveband BEC (red curves), we see that $n_{\mathrm{ex}}$ vanishes when the

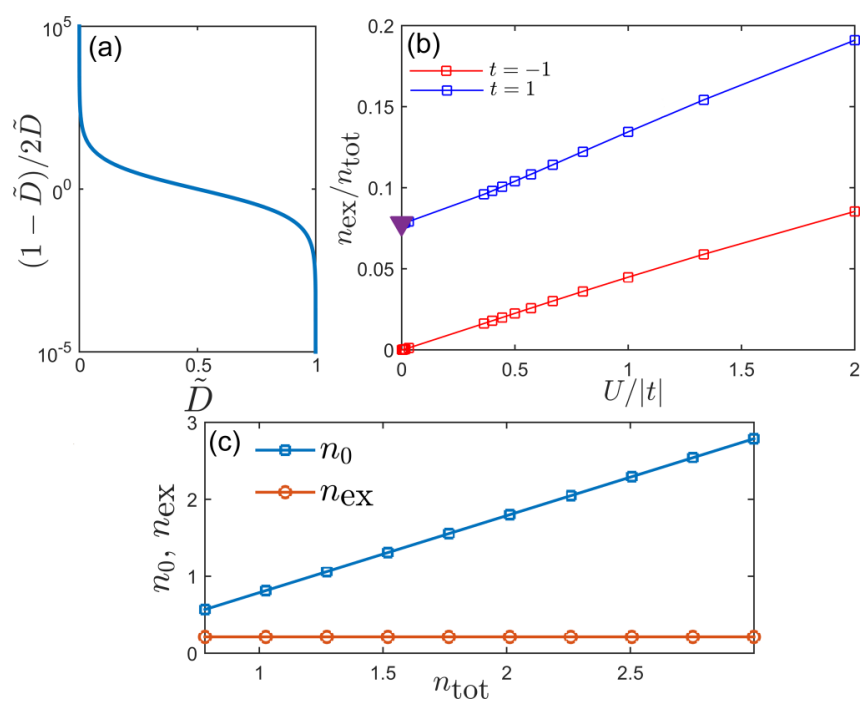

FIG. 4. Excitation density. (a) Functional form of Eq. (20). For $\tilde{D} \rightarrow 0$, one has $(1-\tilde{D}) / 2 \tilde{D} \rightarrow \infty$, which implies instability of the BEC and breakdown of the Bogoliubov theory. (b) Excitation fraction $n_{\mathrm{ex}} / n_{\mathrm{tot}}$ of the two kagome lattice condensation schemes for $n_{\text {tot }}=3$ as a function of $U$. For the flat-band condensate $(t=1)$, $n_{\text {ex }}$ remains finite even in the limit of $U \rightarrow 0$. Purple triangle depicts the analytical result of Eq. (20). (c) Densities $n_{0}$ and $n_{\mathrm{ex}}$ as a function of $n_{\text {tot }}$ for kagome flat-band condensation at $\mathbf{k}_{c}=[4 \pi / 3,0]$ and $U=|t| / 1800$. Excitation density $n_{\mathrm{ex}}$ remains constant, as it is determined by only the quantum geometry.

interaction goes to zero as usual. In contrast, for the flat-band condensate $n_{\mathrm{ex}}$ is nonzero when $U \rightarrow 0$, indeed approaching the value obtained by integrating Eq. (20) over the BZ (purple triangle). The different behavior of $n_{\mathrm{ex}}$ for dispersive- and flat-band condensates is summarized in Fig. 1.

In Fig. 5(a), we present $n_{\text {ex }}(\mathbf{k})$ of the kagome flat-band BEC across the BZ for a small $U$. As predicted by Eq. (20), the momentum dependence of $\tilde{D}(\mathbf{k})$, shown in Fig. 3(c), is imprinted to the momentum distribution of $n_{\mathrm{ex}}(\mathbf{k})$. Similar conclusions can be reached by considering the CB-III model [Fig. 5(b)], where $n_{\mathrm{ex}}(\mathbf{k})$ is nonzero only near the momenta for which $\tilde{D}(\mathbf{k})$ vanishes [see Fig. 3(d)].

The kagome flat-band condensate respects the condition $\left|u_{1}^{*}(\mathbf{k})\right\rangle=\left|u_{1}(\mathbf{k})\right\rangle$ and therefore $\tilde{D}(\mathbf{q})$ reduces to the usual Hilbert-Schmidt quantum distance $D(\mathbf{q})$ (13). Thus, in the kagome lattice, the Hilbert-Schmidt distance of the flat-band states directly determines $n_{\mathrm{ex}}$. The Hilbert-Schmidt distance has been previously connected to Landau level spreading in noninteracting flat-band models [15], and our result reveals how the quantum distance affects physically relevant quantities in an interacting many-body quantum system.

Remarkably, $\lim _{U \rightarrow 0} n_{\text {ex }}$ of the flat-band BEC in Eq. (20) does not depend on $n_{\text {tot }}$ but is determined by the quantum distance. Therefore, one can, by decreasing $n_{\text {tot }}$, increase the excitation fraction $n_{\mathrm{ex}} / n_{\mathrm{tot}}$ and the role of the interaction and quantum fluctuation effects even in the noninteracting limit $U \rightarrow 0$. We show this in Fig. 4(c) where $n_{0}$ and $n_{\mathrm{ex}}$ are depicted as a function of $n_{\text {tot }}$ for small interaction of $U n_{0} / E_{g}=$ $5.13 \times 10^{-4}$ in the case of the kagome model. The excitation density $n_{\text {ex }}$ remains constant as a function of $n_{\text {tot }}$, in contrast to $n_{0}$ which decreases when the total density becomes smaller. 

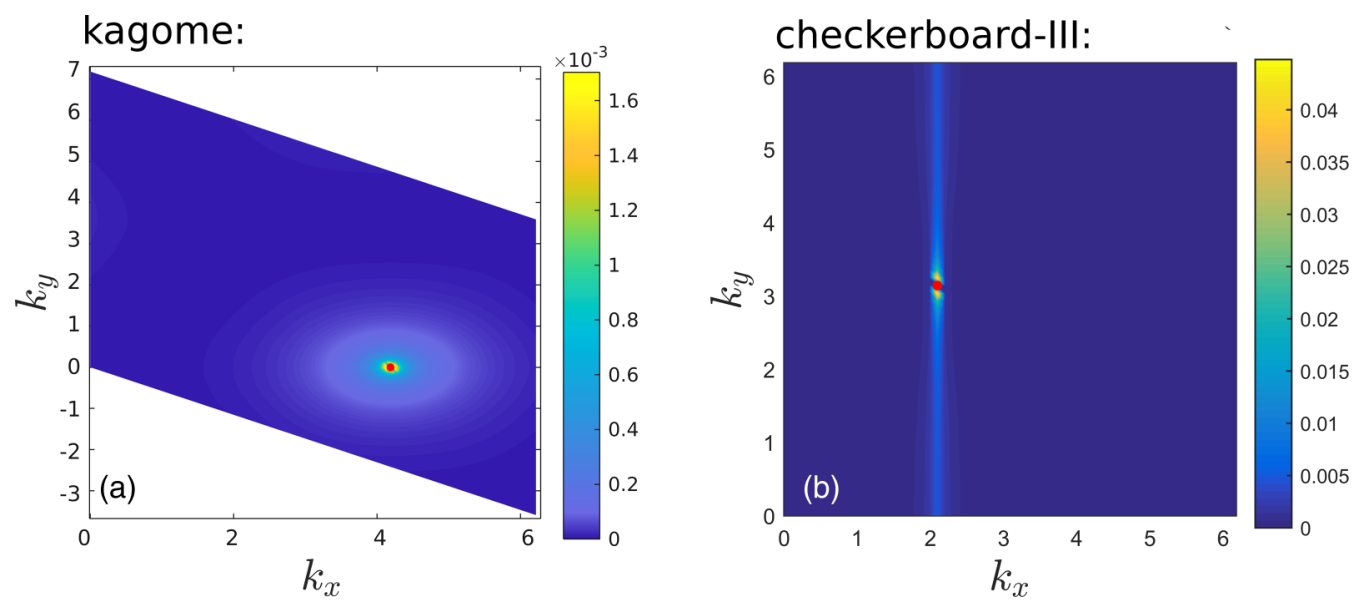

FIG. 5. Momentum dependence of the excitation density. (a), (b) Momentum dependence of $n_{\mathrm{ex}}(\mathbf{k})$ for kagome and CB-III lattices, respectively, with $n_{\text {tot }}=M$. The interaction in the kagome (CB-III) model is chosen to be $U n_{0} / E_{g}=5.13 \times 10^{-4}\left(U n_{0} / E_{g}=0.011\right)$; in the case of CB-III, we use $\delta=10^{-5}$ to avoid a diverging $n_{\text {ex }}$. Remarkably, the excitation fraction follows the features of $\tilde{D}$ shown in Figs. 3(c) and 3(d), as predicted by Eq. (20).

Note that in Fig. 4(c) we consider the regime $n_{\mathrm{ex}} \sim n_{0}$ and thus one might anticipate the usual Bogoliubov theory to break down [70]. We thus also considered the Hartree-FockBogoliubov (HFB) approximation, which, unlike the simple Bogoliubov theory, takes into account the first-order selfenergy diagrams not containing any condensate propagators [see Appendix E and Fig. S1 in the Supplemental Material (SM) [71]]. Even for rather large $n_{\mathrm{ex}} / n_{\mathrm{tot}}$, the Bogoliubov and HFB approximations yield the same results in the limit of $U \rightarrow 0$. This agreement between HFB and Bogoliubov theory confirms the accuracy of our approach, even when $n_{\text {ex }}$ becomes rather large.

The lowest total density used in Fig. 4(c) is approximately $n_{\text {tot }} \sim 0.75$. By utilizing the HFB approach for even smaller total densities, one notes that it is unable to yield self-consistent solutions, implying that our assumption about the existence of a homogeneous BEC might become invalid. It was shown in Ref. [56] using mean-field theory that for densities $n_{\text {tot }} \lesssim 0.72$, a charge density wave (CDW) coexists, alongside the condensate at $\mathbf{k}_{c}=[4 \pi / 3,0]$. Therefore, the breakdown of our HFB method around this density may occur because of a coexisting competing order which is not taken into account in the HFB theory. In Ref. [56], the coexistence of the CDW order was confirmed by exact diagonalization computations.

The dilute limit considered in Ref. [56] sets important limits for the applicability of our Bogoliubov and HFB approach, that assume the existence of a BEC. Namely, it can easily be shown analytically [56] that for densities $n_{\text {tot }} \leqslant \frac{1}{3}$, the ground state consists of spatially nonoverlapping compact localized states (CLS), leading to CDW order. This is a unique feature of flat-band systems since CLS states can be constructed as superpositions of flat-band states only [69,72]. Below the critical density $n_{\mathrm{tot}, c}=\frac{1}{3}$, the particles are distributed among spatially nonoverlapping CLSs. However, for $n_{\mathrm{tot}} \geqslant n_{\mathrm{tot}, c}$ it is not (due to the repulsive interaction) energetically favorable to accumulate particles only to the nonoverlapping CLSs and added bosons hop between interstitial sites of the CDW order, forming a condensate at $\mathbf{k}_{c}=[4 \pi / 4,0]$. In Ref. [56], the di- lute densities of $n_{\mathrm{tot}} \in\left[\frac{1}{3}, \sim 0.72\right]$ were studied showing that both the CDW and the BEC phases can exist or coexist such that the order parameter of CDW (BEC) decreases (increases) as a function of $n_{\text {tot }}$. In this work, however, we are interested in BEC phenomena of flat-band systems rather than different coexisting many-body phases, that possibly take place within a flat-band system. Accordingly, we do not consider the dilute density limit of Ref. [56] but instead higher densities. While our Bogoliubov and HFB theories ignore a possible coexisting CDW phase, it is expected that our method should capture the essential physical features of the BEC phase itself. Based on Ref. [56], the BEC is expected to be favorable as compared to the CDW order at higher densities. Our calculations are therefore reliable for the kagome lattice system at the densities we consider.

\section{SECOND-ORDER CORRELATIONS}

We further unravel the significance of the fluctuations by considering the second-order coherence $g^{(2)}(\mathbf{r}, \tau)$ where $\mathbf{r}$ is the separation of two spatial positions and $\tau$ of two time instances. Specifically, we focus on the correlations within a unit cell:

$$
\begin{aligned}
g^{(2)}(0,0) & =\frac{\frac{1}{N} \sum_{i \alpha \beta}\left\langle c_{i \alpha}^{\dagger} c_{i \beta}^{\dagger} c_{i \alpha} c_{i \beta}\right\rangle}{n_{\mathrm{tot}}^{2}} \\
& =\frac{\frac{1}{N} \sum_{i}\left\langle n_{i} n_{i}\right\rangle-n_{\mathrm{tot}}}{n_{\mathrm{tot}}^{2}},
\end{aligned}
$$

where the second form follows from the bosonic commutation rules. Here $n_{i}=\sum_{\alpha} c_{i \alpha}^{\dagger} c_{i \alpha}$ is the density operator of the $i$ th unit cell. In other words, we are interested in the sum of second-order coherences within a unit cell, averaged over all the unit cells of the lattice.

By treating Eq. (21) at the Bogoliubov level, one finds (see SM [71] for details) that the resulting $g^{(2)}$ consists of three terms, i.e., $g^{(2)}=\sum_{i=1}^{3} g_{i}^{(2)}$. The first term is the MF contribution, reading as $g_{1}^{(2)}=1-1 / n_{\text {tot }}$, whereas $g_{2}^{(2)}$ involves the overlap functions between the Bogoliubov excitations and the 


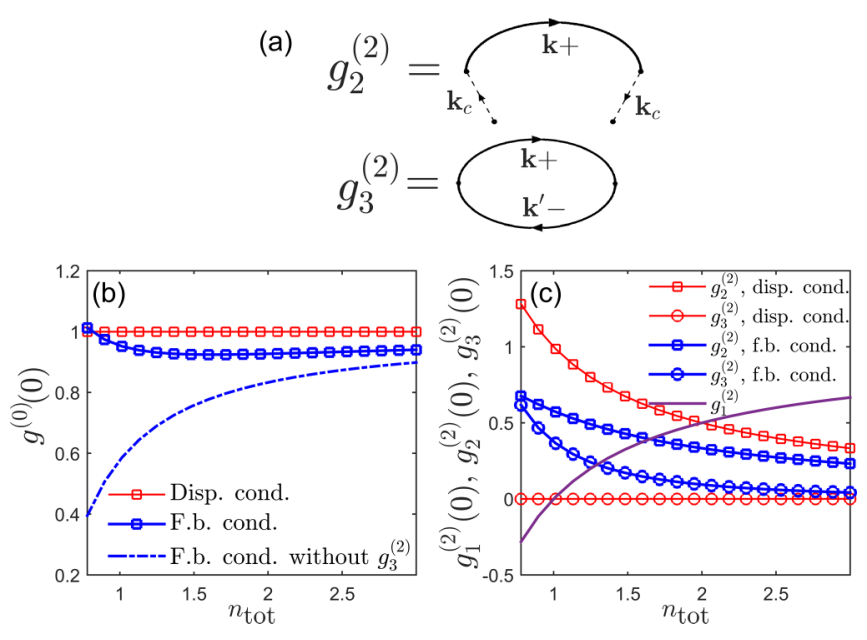

FIG. 6. Second-order local correlator. (a) Diagrammatic presentation of $g_{2}^{(2)}$ and $g_{3}^{(2)}$. The solid (dashed) lines depict the Bogoliubov (condensate) propagators. (b) The second-order correlation function $g^{(2)}$ for the kagome lattice in the case of dispersive- (red curve) and flat-band (blue curve) condensates. The dashed blue line gives $g_{1}^{(2)}+g_{2}^{(2)}$ for the flat-band condensate. (c) Terms $g_{2}^{(2)}$ and $g_{3}^{(2)}$ for the dispersive-band condensate (red curves) and flat-band condensate (blue curves) as a function of $n_{\mathrm{tot}}$. The term $g_{1}^{(2)}$ is also shown (purple curve) and is the same for both of the cases, as it depends only on $n_{\text {tot }}$. The interaction is set to $U=|t| / 1800$.

condensed Bloch state. Finally, $g_{3}^{(2)}$ describes the contribution arising solely from the quantum fluctuations. The expressions for $g_{2}^{(2)}$ and $g_{3}^{(2)}$ are provided in SM [71] and their Feynman diagrams are depicted in Fig. 6(a).

We find that as $n_{\mathrm{ex}}, g_{3}^{(2)}$ of the flat-band condensate at $U \rightarrow$ 0 is also governed by quantum geometry only. Namely, by considering again only the flat-band degrees of freedom, one finds

$$
\begin{aligned}
\lim _{U \rightarrow 0} g_{3}^{(2)} & =\frac{1}{8 N^{2} n_{\text {tot }}^{2}}\left(\sum_{\mathbf{k}} \frac{\alpha(\mathbf{k})}{\tilde{D}(\mathbf{k})} \sum_{\mathbf{k}} \frac{\alpha^{*}(\mathbf{k})}{\tilde{D}(\mathbf{k})}\right. \\
& \left.+\sum_{\mathbf{k}} \frac{1-\tilde{D}(\mathbf{k})}{\tilde{D}(\mathbf{k})} \sum_{\mathbf{k}} \frac{1+\tilde{D}(\mathbf{k})}{\tilde{D}(\mathbf{k})}\right)
\end{aligned}
$$

This expression diverges (vanishes) when $\tilde{D} \rightarrow 0(\tilde{D} \rightarrow 1)$ and is finite for $0<\tilde{D}<1$. Quantum geometry therefore guarantees finite quantum fluctuations even in the noninteracting limit of $U \rightarrow 0$.

The fluctuation term $g_{3}^{(2)}$ can be made significant by tuning down the total density $n_{\text {tot }}$. This is demonstrated for the kagome lattice in Fig. 6(c), where $g_{2}^{(2)}$ and $g_{3}^{(2)}$ are shown as a function of $n_{\text {tot }}$ for flat- and dispersive-band condensates for a small interaction of $U=|t| / 1800$. We see $g_{3}^{(2)}$ being negligible for the dispersive-band condensate, whereas quantum geometry guarantees finite $g_{3}^{(2)}$ for the flat-band condensate. For decreasing $n_{\text {tot }}$, the fluctuation term $g_{3}^{(2)}$ increases and eventually becomes comparable to $g_{2}^{(2)}$. The full coherence function in Fig. 6(b) shows essentially the coherent BEC value $g^{(2)}=1$ in the dispersive case, as expected. Intriguingly, the flat-band condensate shows antibunching behavior, despite the minute value of the interaction $U$, which crosses over to
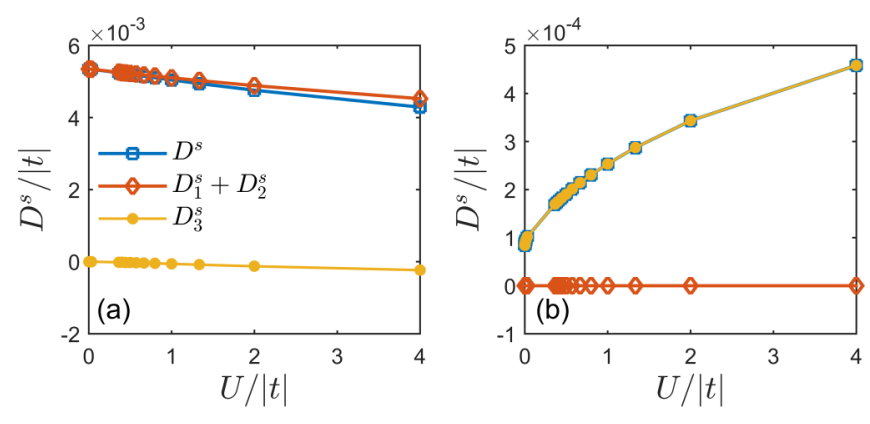

FIG. 7. Zero-temperature superfluid weight $D^{s}$ and its different components for the kagome lattice as a function of $U$. (a) Dispersiveband condensate. (b) Flat-band condensate. The particle density is set to $n_{\text {tot }}=3$. Superfluidity of the flat band is governed by the fluctuation term $D_{3}^{s}$ alone.

the bunching regime at small densities. This nonmonotonic behavior as a function of the density could be used experimentally to probe the effect of quantum geometry in case of a flat-band condensate. The fundamental difference between the second-order coherence function for dispersive- and flat-band BECs is outlined in Fig. 1.

\section{SUPERFLUID WEIGHT}

Finite $c_{s}$ guarantees the possibility of superfluidity, but to understand the phenomenon more deeply, we consider the superfluid weight tensor $D_{\mu \nu}^{s}$ (also referred to as superfluid density or superfluid stiffness in the literature). The superfluid weight is the long-wavelength, zero-frequency limit of the current-current linear response $K_{\mu \nu}(\mathbf{q}, \omega)$ [12], i.e.,

$$
D_{\mu \nu}^{s}=\lim _{\mathbf{q} \rightarrow 0} \lim _{\omega \rightarrow 0} K_{\mu \nu}(\mathbf{q}, \omega) .
$$

We calculate this by deploying the Matsubara Green's function formalism in the framework of the Bogoliubov theory (see SM [71]) at zero temperature. The resulting $D^{s}$ can be divided into three contributions, i.e., $D_{\mu \nu}^{s}=\sum_{i=1}^{3} D_{, i \mu \nu}^{s}$. The first term is the pure condensate contribution and reads as $D_{1, \mu \nu}^{s}=n_{0}\left\langle\phi_{0}\left|\partial_{\mu} \partial_{\nu} \mathcal{H}\left(\mathbf{k}_{c}\right)\right| \phi_{0}\right\rangle$. This generalizes the usual mean-field result of a single-band system $D^{s}=n_{0} / m_{\text {eff }}$, where the effective mass $m_{\text {eff }}$ is replaced by $\left\langle\phi_{0}\left|\partial_{\mu} \partial_{\nu} \mathcal{H}\left(\mathbf{k}_{c}\right)\right| \phi_{0}\right\rangle$ accounting for the multiband nature of the system. The second term $D_{2}^{s}$ constitutes the mixed contribution of the condensate wave function and quantum fluctuations, and the third term $D_{3}^{s}$ arises solely from the quantum fluctuations, i.e., from the Bogoliubov excitations. The expressions for $D_{2}^{s}$ and $D_{3}^{s}$ are provided in SM [71].

For a single-band square lattice, one has $D_{1, \mu \mu}^{s}=$ $n_{0} \partial_{\mu}^{2} \epsilon_{1}\left(\mathbf{k}_{c}\right), D_{2}^{s}=0$, and $D_{3}^{s}<0$. Thus, the condensation contribution $D_{1}^{s}$ is determined by the inverse effective mass of bosons at $\mathbf{k}_{c}$ and quantum fluctuations decrease the supercurrent via negative $D_{3}^{s}$. However, for a flat-band condensate, the story is very different. We demonstrate this in Fig. 7 by depicting $D^{s}$ and its components as a function of $U$ in the case of the kagome model for both the dispersive- [Fig. 7(a)] and flat-band condensates [Fig. 7(b)]. For the dispersive-band condensate, $D^{s}$ is mainly given by $D_{1}^{s}+D_{2}^{s}$ and the fluctuation term $D_{3}^{s}$ gives a negative contribution. In stark contrast, the 
flat-band condensate results depicted in Fig. 7(b) reveal that $D^{s}$ is solely determined by the fluctuation term $D_{3}^{s}$. Therefore, instead of inhibiting the superfluidity, quantum fluctuations are actually responsible for finite superfluidity of the flat-band condensate.

Flat-band superfluidity is governed by $D_{3}^{s}$ because $D_{2}^{s}$ cancels the condensate contribution $D_{1}^{s}$. This can be proven in the weak-coupling limit $U \rightarrow 0$ (shown in SM [71]). For the kagome lattice, the cancellation also takes place for finite interactions as shown in Fig. 7(b). A very similar result was obtained recently in Ref. [73], where condensation was studied in a two-dimensional spin-orbit coupled system featuring a one-particle dispersion with a continuous subset of degenerate ground states on a circle of equal momentum amplitudes. Also in that case, finite superfluidity in the direction where the effective mass diverges was shown to arise from the quantum fluctuations only.

Our result regarding quantum fluctuation-mediated flatband superfluidity is intuitively easy to understand. Namely, due to destructive interference among the flat-band states, bosons condensed to a single-flat-band Bloch state are spatially localized to a subset of lattice sites and thus have vanishing group velocity. In contrast, for a dispersive-band $\mathrm{BEC}$, it is the condensate that mainly carries the supercurrent as the effective mass of a dispersive-band BEC is finite. In case of a flat-band BEC, instead, it is the fluctuations, arising due to interactions, that are needed for a finite supercurrent. This fundamental difference regarding the origin of superfluidity in disperisve- and flat-band condensates is summarized in Fig. 1.

The fluctuation term $D_{3}^{s}$ is similar to the superfluid weight of fermionic systems $[10,12]$, except for factors accounting for fermionic statistics, as expected from the formal connection between bosonic Bogoliubov and fermionic BCS approximations. Like fermionic $D^{s}, D_{3}^{s}$ can also be split into intraband ("conventional") and interband ("geometric") terms, i.e., $D_{3}^{s}=D_{3 \text {,conv }}^{s}+D_{3 \text {,geom }}^{s}$. Specifically, $D_{3}^{s}$ contains the current terms of the form (see SM [71])

$$
\begin{aligned}
&\left\langle u_{n}(\mathbf{k})\left|\partial_{\mu} \mathcal{H}(\mathbf{k})\right| u_{m}(\mathbf{k})\right\rangle=\partial_{\mu} \epsilon_{n}(\mathbf{k}) \delta_{m n} \\
&+\left[\epsilon_{m}(\mathbf{k})-\epsilon_{n}(\mathbf{k})\right]\left\langle\partial_{\mu} u_{n}(\mathbf{k}) \mid u_{m}(\mathbf{k})\right\rangle .
\end{aligned}
$$

The intraband term $D_{3 \text {,conv }}^{s}$ contains only the diagonal current terms $(m=n)$ proportional to the derivatives of the single-particle dispersions $\partial_{\mu} \epsilon_{n}(\mathbf{k})$, whereas the geometric contribution $D_{3, \text { geom }}^{s}$ includes the interband current terms $(m \neq n)$ proportional to the interband Berry connection $\left\langle\partial_{\mu} u_{n}(\mathbf{k}) \mid u_{m}(\mathbf{k})\right\rangle$. For a flat band $D_{3, \text { conv }}^{s}$ vanishes due to momentum-independent dispersion, and $D_{1}^{s}$ and $D_{2}^{s}$ cancel each other, therefore, it is the geometric part of the fluctuation term $D_{3, \text { geom }}^{s}$ that is responsible for the superfluidity of the flat-band condensate.

\section{CONCLUSION}

We have deployed multiband Bogoliubov theory to study how quantum geometric properties of the Bloch states affect various physical quantities of a weakly interacting BEC, with the emphasis being in lattice models that feature flat bands. We showed that in case of a flat-band BEC, the speed of sound $c_{s}$ is dictated by the quantum metric and the excitation density $n_{\mathrm{ex}}$ remains finite at $U \rightarrow 0$ due to finite quantum distance between the condensed state and other Bloch states of the band. Similarly, we demonstrated that also the quantum fluctuation part of the density-density correlations remains finite in the limit of $U \rightarrow 0$ and is given by the same quantum distance.

Our results demonstrate the unusually prominent role of fluctuations in a flat-band BEC. The weak-coupling limit of the excitation fraction and the quantum fluctuation contribution of the density-density correlator do not depend on the condensate density, and therefore their relative importance can be enhanced by reducing the total density. Even the extreme situation where the superfluid weight is given by the quantum fluctuations alone can be achieved in the small interaction limit.

The fact that interactions, quantum fluctuations, and correlation effects may become enhanced has been a key motivation for studies of flat bands. Our work shows that indeed this promise can be fulfilled in the case of bosonic condensates, and that these effects can be controlled by the underlying nontrivial quantum geometry, that is, a nonzero quantum metric, and distances between the Bloch states. A nontrivial quantum geometry guarantees the stability of the condensate via a nondiverging excitation fraction, and finite speed of sound. Furthermore, it allows to realize situations where quantum fluctuations and correlations dominate the system behavior.

Our work, alongside the accompanying study in Ref. [64], establishes important connections between the quantum geometry and physical quantities of a BEC, showing how the geometric properties of quantum states can be utilized to reach the strongly correlated regime. This can have important consequences, especially in systems where the interactions between particles are inherently small compared to kinetic tunneling energies and quantum correlations and depletion are weak, e.g., in photonic or polaritonic platforms. It could provide a key step towards realizing strongly correlated photons, important for both fundamental research and information processing. In long-lived, quasiequilibrium photon and polariton systems our results should directly apply, while extending them to the strongly driven-dissipative case is an interesting future research task.

\section{ACKNOWLEDGMENTS}

We thank L. Liang and M. Iskin for useful discussions. A.J. and P.T. acknowledge support by the Academy of Finland under Projects No. 303351, No. 307419, and No. 327293 A.J. acknowledges financial support from the Jenny and Antti Wihuri Foundation. This research was supported in part by the National Science Foundation under Grant No. PHY-1748958. We thank A. Paraoanu for producing the illustrations for Fig. 1.

\section{APPENDIX A: MEAN-FIELD APPROXIMATION}

To solve the Bloch momentum state in which the Bose condensate takes place, we utilize the mean-field (MF) approximation where we substitute operators in Eq. (2) 
by complex numbers, i.e., $c_{\mathbf{k}} \rightarrow \psi_{\mathbf{k}} \equiv\left[\psi_{\mathbf{k} 1}, \ldots, \psi_{\mathbf{k} M}\right]^{T}$ and minimize the resulting MF energy

$$
E_{\mathrm{MF}}(\mathbf{k})=\psi_{\mathbf{k}}^{\dagger} \mathcal{H}(\mathbf{k}) \psi_{\mathbf{k}}+\frac{U}{2} \sum_{\alpha}\left|\psi_{\mathbf{k}, \alpha}\right|^{4}
$$

for each $\mathbf{k}$ separately with respect to the constraint $\sum_{\alpha}\left|\psi_{\alpha}\right|^{2}=n_{0} \quad$ [62]. The constraint ensures that the condensate density $n_{0}$ is the same for all $\mathbf{k}$. The condensation is then chosen to take place at the Bloch momentum $\mathbf{k}_{c}$ and state $\left|\phi_{0}\right\rangle$ for which the MF energy $E_{\mathrm{MF}}(\mathbf{k})$ is minimized, i.e.,

$$
\begin{aligned}
E_{\mathrm{MF}}\left(\mathbf{k}_{c}\right) & =\min \left[E_{\mathrm{MF}}(\mathbf{k})\right] \\
& =\left\langle\phi_{0}\left|\mathcal{H}\left(\mathbf{k}_{c}\right)\right| \phi_{0}\right\rangle+\frac{U}{2} \sum_{\alpha}\left|\left\langle\alpha \mid \phi_{0}\right\rangle\right|^{4}
\end{aligned}
$$

with $\left\langle\alpha \mid \phi_{0}\right\rangle$ being the projection of $\left|\phi_{0}\right\rangle$ to orbital $\alpha$. Thus, even though the flat band is strictly flat, the interaction term can favor a subset of Bloch states for which the condensate density is distributed as uniformly as possible between the sublattices to minimize the repulsive onsite interaction. For example, in the case of the kagome lattice, the condensed state at $\mathbf{k}_{c}=[4 \pi / 3,0]$ reads as $\phi_{0}=[-1,-1,1] / \sqrt{3}$ whose sublattice density $\left|\left\langle\alpha \mid \phi_{0}\right\rangle\right|^{2}=\frac{1}{3}$ is the same for all three sublattices. Similarly, in the case of the checkerboard-III flat band, the condensed state at $\mathbf{k}_{c}=[2 \pi / 3, \pi]$ is $\phi_{0}=[i, 1]$, yielding uniform sublattice density $\left|\left\langle\alpha \mid \phi_{0}\right\rangle\right|^{2}=\frac{1}{2}$.

\section{APPENDIX B: DETAILS ON BOGOLIUBOV APPROXIMATION}

Here we outline the the well-known Bogoliubov theory $[63,70]$ for a multiband system. To this end, one defines the bosonic Green's function for the noncondensed bosons in the imaginary-time domain as follows:

$$
\begin{aligned}
& G(\mathbf{k}, \tau) \equiv-\left\langleT _ { \tau } [ \begin{array} { c } 
{ c _ { \mathbf { k } } ( \tau ) } \\
{ c _ { 2 \mathbf { k } _ { c } - \mathbf { k } } ^ { \dagger } ( \tau ) }
\end{array} ] \left[\begin{array}{ll}
c_{\mathbf{k}}^{\dagger}(0) & \left.\left.c_{2 \mathbf{k}_{c}-\mathbf{k}}(0)\right]\right\rangle
\end{array}\right.\right. \\
& =\left[\begin{array}{cc}
-\left\langle T_{\tau} c_{\mathbf{k}}(\tau) c_{\mathbf{k}}^{\dagger}(0)\right\rangle & -\left\langle T_{\tau} c_{\mathbf{k}}(\tau) c_{2 \mathbf{k}_{c}-\mathbf{k}}(0)\right\rangle \\
-\left\langle T_{\tau} c_{2 \mathbf{k}_{c}-\mathbf{k}}^{\dagger}(\tau) c_{\mathbf{k}}^{\dagger}(0)\right\rangle & -\left\langle T_{\tau} c_{2 \mathbf{k}_{c}-\mathbf{k}}^{\dagger}(\tau) c_{2 \mathbf{k}_{c}-\mathbf{k}}(0)\right\rangle
\end{array}\right] \\
& \equiv\left[\begin{array}{ll}
G_{11}(\mathbf{k}, \tau) & G_{12}(\mathbf{k}, \tau) \\
G_{21}(\mathbf{k}, \tau) & G_{22}(\mathbf{k}, \tau)
\end{array}\right] \text {. }
\end{aligned}
$$

Here, $T_{\tau}$ is the imaginary-time ordering operator, $\tau$ is imaginary time, and $\mathbf{k} \neq \mathbf{k}_{c}$ as we consider the noncondensed bosons. Note that in our multiband case, each block $G_{i j}(\mathbf{k}, \tau)$ in (B1) is a $M \times M$ matrix. In the bosonic Matsubara frequency space, one has the Dyson equation $[63,70]$

$$
\begin{aligned}
G^{-1}\left(\mathbf{k}, i \omega_{n}\right)= & G_{0}^{-1}\left(\mathbf{k}, i \omega_{n}\right)-\Sigma\left(\mathbf{k}, i \omega_{n}\right) \\
& \Leftrightarrow\left[\begin{array}{cc}
G_{11}(\mathbf{k}, i \omega) & G_{12}(\mathbf{k}, i \omega) \\
G_{21}(\mathbf{k}, i \omega) & G_{22}(\mathbf{k}, i \omega)
\end{array}\right]^{-1} \\
= & {\left[\begin{array}{cc}
G_{0,11}(\mathbf{k}, i \omega) & 0 \\
0 & G_{0,22}(\mathbf{k}, i \omega)
\end{array}\right] } \\
& -\left[\begin{array}{cc}
\Sigma_{11}(\mathbf{k}, i \omega) & \Sigma_{12}(\mathbf{k}, i \omega) \\
\Sigma_{21}(\mathbf{k}, i \omega) & \Sigma_{22}(\mathbf{k}, i \omega)
\end{array}\right] .
\end{aligned}
$$

Here, $\omega_{n}$ is a bosonic Matsubara frequency and $\Sigma$ is the selfenergy arising from the finite interaction $U \neq 0$ and $G_{0}$ is the noninteracting Green's function which reads as

$$
G_{0}^{-1}\left(\mathbf{k}, i \omega_{n}\right)=i \omega_{n} \sigma_{z}-\left[\begin{array}{cc}
\mathcal{H}(\mathbf{k})-\mu & 0 \\
0 & \mathcal{H}^{*}(\mathbf{k})-\mu
\end{array}\right] .
$$

Here, $\sigma_{z}$ is the Pauli matrix in the particle-hole basis. In general, the self-energy $\Sigma\left(\mathbf{k}, i \omega_{n}\right)$ is evaluated by using the diagrammatic Beliaev theory $[63,70]$. The Bogoliubov theory is the first-order approximation of the Belieav approach [70], containing the self-energy diagrams illustrated in black color in Fig. S1 in the SM [71]. Specifically, the Bogoliubov theory includes only the first-order diagrams that contain the condensate propagators. In the case of a momentum-independent contact interaction, it is straightforward to evaluate both the diagonal and off-diagonal self-energy blocks $\Sigma_{11}$ and $\Sigma_{12}$ :

$$
\begin{gathered}
{\left[\Sigma_{11}(\mathbf{k})\right]_{\alpha \beta}=\delta_{\alpha \beta} 2 U n_{0}\left|\left\langle\alpha \mid \phi_{0}\right\rangle\right|^{2},} \\
{\left[\Sigma_{12}(\mathbf{k})\right]_{\alpha \beta}=\delta_{\alpha \beta} U n_{0}\left\langle\alpha \mid \phi_{0}\right\rangle^{2} .}
\end{gathered}
$$

We assume uniform condensate such that $\left|\left\langle\alpha \mid \psi_{0}\right\rangle\right|^{2}=1 / M$. The full Green's function is then obtained from the Dyson equation (B2):

$$
\begin{aligned}
& G^{-1}\left(\mathbf{k}, i \omega_{n}\right)=i \omega_{n} \sigma_{z} \\
& \quad-\left[\begin{array}{cc}
\mathcal{H}(\mathbf{k})-\mu+2 \frac{U n_{0}}{M} & \Delta \\
\Delta^{*} & \mathcal{H}^{*}\left(2 \mathbf{k}_{c}-\mathbf{k}\right)-\mu+2 \frac{U n_{0}}{M}
\end{array}\right] .
\end{aligned}
$$

Here, $[\Delta]_{\alpha \beta}=\delta_{\alpha, \beta} U n_{0}\left\langle\alpha \mid \phi_{0}\right\rangle^{2}$. By rewriting $\mathbf{k}=\mathbf{k}_{c}+\mathbf{q}$, we see that the upper (lower) diagonal block in (B6) represents particles (holes) traveling with momentum $\mathbf{q}(-\mathbf{q})$ with respect to the momentum $\mathbf{k}_{c}$ of the condensate.

The poles of $G\left(\mathbf{k}, i \omega_{n}\right)$ give the excitation spectrum of the Bose condensed system [63]. As we are dealing with the equilibrium condensate, the lowest excitation band has to be gapless at $\mathbf{k}_{c}$, i.e., a pole must exist at $\omega=0$ for $\mathbf{k}=\mathbf{k}_{c}$ [70]. For our multiband system, we find that this condition forces $\mu$ to satisfy the following expression:

$$
\begin{aligned}
& \epsilon_{0}-\mu+\frac{2 U n_{0}}{M}-\frac{U^{2} n_{0}}{M^{2}}\left(\epsilon_{0}-\mu+\frac{2 U n_{0}}{M}\right)^{-1}=0 \\
& \Leftrightarrow \mu=\epsilon_{0}+\frac{U n_{0}}{M} .
\end{aligned}
$$

This is the multiband generalization of the usual HugenholtzPines relation $[63,70]$. We should note that for obtaining Eq. (B7), the uniform condensation assumption $\left|\left\langle\alpha \mid \phi_{0}\right\rangle\right|^{2}=$ $1 / M$ is crucial. If this was not the case, solving $\mu$ would most likely require numerical evaluation.

By combining the expression of $\mu$ to Eq. (B6), one gets

$$
G^{-1}\left(\mathbf{k}, i \omega_{n}\right)=i \omega_{n} \sigma_{z}-\left[\begin{array}{cc}
\mathcal{H}(\mathbf{k})-\mu_{\mathrm{eff}} & \Delta \\
\Delta^{*} & \mathcal{H}^{*}\left(2 \mathbf{k}_{c}-\mathbf{k}\right)-\mu_{\mathrm{eff}}
\end{array}\right],
$$

where $\mu_{\text {eff }}=\epsilon_{0}-\frac{U n_{0}}{M}$. The Bogoliubov approximation yields a quadratic Hamiltonian, and it is easy to show that $G^{-1}\left(\mathbf{k}, i \omega_{n}\right)=i \omega_{n} \sigma_{z}-\mathcal{H}_{B} \quad$ so that $\mathcal{H}_{B}(\mathbf{k})=-G^{-1}(\mathbf{k}, 0)$. This is the Bogoliubov Hamiltonian expressed in Eq. (4).

In our numerical Bogoliubov calculations, we fixed the total density $n_{\text {tot }}$ and chose an initial ansatz for $n_{0}$. This was then substituted to $L(\mathbf{k})=\sigma_{z} \mathcal{H}_{B}(\mathbf{k})$ and the Bogoliubov states 
$\left|\psi_{m}^{ \pm}(\mathbf{k})\right\rangle$ were obtained by carrying out the diagonalization of Eq. (6). To ensure the Bogoliubov states follow the bosonic commutation rules, we demand the standard normalization condition for the Bogoliubov states to hold [66]:

$$
\begin{aligned}
& { }_{1}\left\langle\psi_{m}^{ \pm}(\mathbf{k}) \mid \psi_{m}^{ \pm}(\mathbf{k})\right\rangle_{1}-{ }_{2}\left\langle\psi_{m}^{ \pm}(\mathbf{k}) \mid \psi_{m}^{ \pm}(\mathbf{k})\right\rangle_{2}= \pm 1, \text { with } \\
& \left|\psi_{m}^{ \pm}(\mathbf{k})\right\rangle \equiv\left[\left|\psi_{m}^{ \pm}(\mathbf{k})\right\rangle_{1},\left|\psi_{m}^{ \pm}(\mathbf{k})\right\rangle_{2}\right]^{T}
\end{aligned}
$$

Based on the obtained Bogoliubov states, $n_{\mathrm{ex}}$ was then calculated with Eq. (19). In this way, a new value for $n_{0}=n_{\mathrm{tot}}-n_{\mathrm{ex}}$ was acquired and then substituted back to $L(\mathbf{k})$. This iteration procedure was continued until a self-consistent solution for $n_{0}$ was found.

In general, Bogoliubov theory is based on the assumption that the system is Bose condensed and the remaining quantum fluctuation effects can be described by the quadratic Bogoliubov Hamiltonian (4). This approximation is based on the assumption that the local onsite interaction energy $U$ times condensate density per unit cell $n_{0}$, i.e., $U n_{0}$, is small compared to the kinetic hopping terms $\mathcal{H}_{i \alpha j \beta}$ of the Hamiltonian of Eq. (1). In our calculations, this is always the case. Furthermore, conventionally the Bogoliubov theory is considered to hold only for cases with $n_{\mathrm{ex}} \ll n_{\mathrm{tot}}$. However, as we discuss at the end of Sec. IV and Appendix E, our method should be still valid even for cases when $n_{\mathrm{ex}}$ is of the same order of magnitude than $n_{\text {tot }}$ as long as the aforementioned condition on $U n_{0} \ll \mathcal{H}_{i \alpha j \beta}$ holds. In this work we present results for two-dimensional systems, but extension to three dimensions is straightforward as the Bogoliubov theory works well there.

\section{APPENDIX C: PROJECTION TO THE LOWEST BAND}

In obtaining our main results, we used the projected $L_{p}(\mathbf{k})$ of Eq. (9) in which only the lowest Bloch band degrees of freedom were retained and the effects of other Bloch bands were discarded. To obtain (9), we must first transform the full $L(\mathbf{k})=\sigma_{z} \mathcal{H}_{B}$ to the Bloch basis that diagonalizes the kinetic energy Hamiltonian $\mathcal{H}(\mathbf{k})$. Specifically, we first write $\mathcal{H}(\mathbf{k})=U(\mathbf{k}) D(\mathbf{k}) U^{\dagger}(\mathbf{k})$, where $D(\mathbf{k})=$ $\operatorname{diag}\left[\epsilon_{1}(\mathbf{k}), \epsilon_{2}(\mathbf{k}), \ldots, \epsilon_{M}(\mathbf{k})\right]$ and the columns of $U(\mathbf{k})$ contain the corresponding Bloch states. We then define the unitary transformation $\mathcal{U}(\mathbf{k})$ as

$$
\mathcal{U}(\mathbf{k})=\left[\begin{array}{cc}
U(\mathbf{k}) & 0 \\
0 & U^{*}\left(2 \mathbf{k}_{c}-\mathbf{k}\right)
\end{array}\right]
$$

By transforming $L(\mathbf{k}) \rightarrow \mathcal{U}^{\dagger}(\mathbf{k}) L(\mathbf{k}) \mathcal{U}(\mathbf{k})$ and moreover writing the momentum as $\mathbf{k}=\mathbf{k}_{c}+\mathbf{q}$, we have in the Bloch basis the following:

$$
L(\mathbf{k})=\left[\begin{array}{cc}
D\left(\mathbf{k}_{c}+\mathbf{q}\right)-\mu_{\mathrm{eff}} & U^{\dagger}\left(\mathbf{k}_{c}+\mathbf{q}\right) \Delta U^{*}\left(\mathbf{k}_{c}-\mathbf{q}\right) \\
-U^{T}\left(\mathbf{k}_{c}-\mathbf{q}\right) \Delta^{*} U\left(\mathbf{k}_{c}+\mathbf{q}\right) & -D\left(\mathbf{k}_{c}-\mathbf{q}\right)+\mu_{\mathrm{eff}}
\end{array}\right]
$$

By only retaining the lowest Bloch band and projecting out all of the other Bloch band degrees of freedom in Eq. (C2), one obtains $L_{p}(\mathbf{k})$ of Eq. (9).

\section{APPENDIX D: DENSITY OF NONCONDENSED PARTICLES}

The expression (19) for the density of noncondensed particles can be derived as follows:

$$
\begin{aligned}
n_{\mathrm{ex}} & =\frac{1}{N} \sum_{\mathbf{k} \alpha}{ }^{\prime}\left\langle c_{\mathbf{k} \alpha}^{\dagger} c_{\mathbf{k} \alpha}\right\rangle \\
& =\frac{1}{2 N} \sum_{\mathbf{k} \alpha}{ }^{\prime}\left[\left\langle c_{\mathbf{k} \alpha}^{\dagger} c_{\mathbf{k} \alpha}\right\rangle+\left\langle c_{\mathbf{k} \alpha} c_{\mathbf{k} \alpha}^{\dagger}\right\rangle-1\right] \\
& =-\frac{\lim _{\tau \rightarrow 0}}{2 N} \sum_{\mathbf{k}}{ }^{\prime} \operatorname{Tr}[G(\mathbf{k}, \tau)+1] \\
& =-\frac{1}{2 N} \sum_{\mathbf{k}}{ }^{\prime}\left\{M+\frac{1}{\beta} \sum_{i \omega_{n}} \operatorname{Tr}\left[G\left(\mathbf{k}, i \omega_{n}\right)\right]\right\} .
\end{aligned}
$$

Here, $\beta=1 /\left(k_{B} T\right)$ with $k_{B}$ the Boltzmann constant. We can proceed by noting that for the quadratic Bogoliubov Hamiltonian, one can write the Green's function with the aid of the Bogoliubov states and energies as

$$
G\left(\mathbf{k}, i \omega_{n}\right)=\sum_{m, s} s \frac{\left|\psi_{m}^{s}(\mathbf{k})\right\rangle\left\langle\psi_{m}^{s}(\mathbf{k})\right|}{i \omega_{n}-s E_{m}\left(\mathbf{k}_{c}+s \mathbf{q}\right)}
$$

Here, $\mathbf{q}=\mathbf{k}-\mathbf{k}_{c}$ and $\left|\psi_{m}^{s}(\mathbf{k})\right\rangle$ are the Bogoliubov states fulfilling Eqs. (6) and (B9). The validity of Eq. (D2) can be confirmed by multiplying it with $G^{-1}\left(\mathbf{k}, i \omega_{n}\right)=i \omega_{n} \sigma_{z}-\mathcal{H}_{B}$. By substituting Eq. (D2) to (D1) and carrying out the trace, one finds at $T=0$

$$
\begin{aligned}
n_{\mathrm{ex}} & =\frac{(-1)}{2 N} \sum_{\mathbf{k}, m}{ }^{\prime}\left\{1+\frac{1}{\beta} \sum_{s, i \omega_{n}} \frac{s\left\langle\psi_{m}^{s}(\mathbf{k}) \mid \psi_{m}^{s}(\mathbf{k})\right\rangle}{i \omega_{n}-s E_{m}\left(\mathbf{k}_{c}+s \mathbf{q}\right)}\right\} \\
& =\frac{1}{2 N} \sum_{\mathbf{k}, m}{ }^{\prime}\left[-1+\sum_{s} s\left\langle\psi_{m}^{s}(\mathbf{k}) \mid \psi_{m}^{s}(\mathbf{k})\right\rangle n_{B}\left[s E_{m}\left(\mathbf{k}_{c}+s \mathbf{q}\right)\right]\right] \\
& =\frac{1}{2 N} \sum_{\mathbf{k}, m}{ }^{\prime}\left[-1+\left\langle\psi_{m}^{-}(\mathbf{k}) \mid \psi_{m}^{-}(\mathbf{k})\right\rangle\right],
\end{aligned}
$$

where $n_{B}(x)=1 /\left(e^{\beta x}-1\right)$ is the Bose-Einstein distribution, and in the last step we have used the fact that at $T=0$ we have $n_{B}(x>0)=0$ and $n_{B}(x<0)=-1$.

It is instructive to consider $n_{\mathrm{ex}}$ in a lattice geometry which respects the time-reversal symmetry, i.e., $\mathcal{H}(\mathbf{k})=\mathcal{H}^{*}(-\mathbf{k})$, with zero-momentum condensate $\mathbf{k}_{c}=0$ and a scalar valued $\Delta$. This special case covers, for example, the usual square lattice, honeycomb lattice, and the kagome lattice with a dispersive band chosen as the lowest Bloch band (i.e., with negative $\mathrm{NN}$ hopping, $t<0$ ). We then find

$$
n_{\mathrm{ex}}=\frac{1}{2 N} \sum_{\mathbf{k}, m}^{\prime}\left[-1+\left|u^{m}(\mathbf{k})\right|^{2}+\left|v^{m}(\mathbf{k})\right|^{2}\right],
$$


where the coherence factors $u^{m}(\mathbf{k})$ and $v^{m}(\mathbf{k})$ are

$$
\begin{aligned}
& u^{m}(\mathbf{k})=\frac{1}{\sqrt{2}} \sqrt{\frac{\tilde{\epsilon}_{m}(\mathbf{k})+U n_{0} / M}{E_{m}(\mathbf{k})}+1}, \\
& v^{m}(\mathbf{k})=-\frac{1}{\sqrt{2}} \sqrt{\frac{\tilde{\epsilon}_{m}(\mathbf{k})+U n_{0} / M}{E_{m}(\mathbf{k})}-1}
\end{aligned}
$$

with the Bogoliubov energies $E_{m}(\mathbf{k})=$ $\sqrt{\tilde{\epsilon}_{m}(\mathbf{k})\left[\tilde{\epsilon}_{m}(\mathbf{k})+2 U n_{0} / M\right]}$ and $\tilde{\epsilon}_{m}(\mathbf{k}) \equiv \epsilon_{m}(\mathbf{k})-\epsilon_{0}$. Note that each Bogoliubov band $m$ depends only on the $m$ th Bloch band. When the lowest Bloch band is not strictly flat $\left(\tilde{\epsilon}_{1} \neq 0\right)$, one has $\lim _{U \rightarrow 0} n_{\mathrm{ex}}=0$ as $u^{m}(\mathbf{k}) \rightarrow 1$ and $v^{m}(\mathbf{k}) \rightarrow 0$. This is consistent with the result shown in Fig. 4 where $\lim _{U \rightarrow 0} n_{\text {ex }}=0$ for the dispersive-band condensate of the kagome lattice. On the other hand, in the case of a flat-band condensate, one finds with Eq. (D4) that $\lim _{U \rightarrow 0} n_{\mathrm{ex}} \rightarrow \infty$, meaning that one can never achieve a stable flat-band BEC with the aforementioned conditions. Indeed, in the case of the kagome flat-band condensate, finite condensate momentum $\mathbf{k}_{c}=[4 \pi, 0]$ breaks the $\mathbf{k}_{c}=0$ condition, implying that Eq. (D4) is no longer valid and one can then find stable BEC within the flat band.

\section{APPENDIX E: HARTREE-FOCK-BOGOLIUBOV APPROXIMATION}

In our calculations we can have $n_{\mathrm{ex}} \sim n_{0}$, and therefore it is not evidently clear whether the Bogoliubov theory is an appropriate method since Bogoliubov theory usually assumes that $n_{0} \gg n_{\mathrm{ex}}$ [70]. It turns out that in the $U \rightarrow 0$ limit (which we are mostly interested in), the Bogoliubov approximation should be a valid choice for our calculations even at the regime of $n_{\mathrm{ex}} \sim n_{0}$. We show this by considering the HartreeFock-Bogoliubov (HFB) theory, which is an extension to the Bogoliubov theory. Specifically, HFB counts for all the firstorder self-energy diagrams shown in Fig. S1 of SM [71]. In addition to the Bogoliubov diagrams, one also accounts the diagrams that do not contain the condensate propagators [70]. By evaluating the diagrams, one finds

$$
\begin{aligned}
{\left[\Sigma_{11}(\mathbf{k})\right]_{\alpha \beta} } & =\delta_{\alpha \beta} 2 U\left(n_{0}\left|\left\langle\alpha \mid \phi_{0}\right\rangle\right|^{2}+n_{\mathrm{ex}, \alpha}\right) \\
& \equiv \delta_{\alpha \beta} 2 U n_{\mathrm{tot}, \alpha}
\end{aligned}
$$

$$
\begin{aligned}
{\left[\Sigma_{12}(\mathbf{k})\right]_{\alpha \beta} } & =\delta_{\alpha \beta} U n_{0}\left\langle\alpha \mid \phi_{0}\right\rangle^{2}+\frac{\delta_{\alpha \beta} U}{N} \sum_{\mathbf{q}}{ }^{\prime}\left\langle c_{\mathbf{q} \alpha} c_{2 \mathbf{k}_{c}-\mathbf{q} \alpha}\right\rangle \\
& \equiv \delta_{\alpha \beta}\left(U n_{0}\left\langle\alpha \mid \phi_{0}\right\rangle^{2}+\Lambda_{\alpha}\right) .
\end{aligned}
$$

Here, $n_{\text {ex }, \alpha}\left(n_{\text {tot }, \alpha}\right)$ is the excitation (total) density of the $\alpha$ th sublattice and solving $\Lambda_{\alpha}$ in $\Sigma_{12}$ requires a self-consistent fixed-point iteration scheme. It is well known that the selfconsistent HFB method has problems with yielding the gapless excitation modes at $\mathbf{k}_{c}=0$ [70,74]. Here we circumvent this inconsistency by explicitly demanding the existence of the gapless Goldstone mode at $\mathbf{k}_{c}$. By assuming uniform density $n_{\text {tot }, \alpha}=n_{\text {tot }} / M$ and furthermore $\Lambda_{\alpha}=\frac{U}{M} \Lambda$, one finds $\mu=\epsilon_{0}+\frac{2 U n_{\text {tot }}}{M}-\frac{U}{M}\left|n_{0}+\Lambda\right|$. We therefore obtain the following quadratic Hamiltonian for the HFB approximation:

$$
\begin{aligned}
H_{\mathrm{HFB}}(\mathbf{k}) & =\left[\begin{array}{cc}
\mathcal{H}_{\mathbf{k}}-\tilde{\mu}_{\mathrm{eff}} & \tilde{\Delta} \\
\tilde{\Delta}^{*} & \mathcal{H}_{\mathbf{k}}^{*}-\tilde{\mu}_{\mathrm{eff}}
\end{array}\right], \\
\tilde{\Delta}_{\alpha \beta} & =[\Delta]_{\alpha \beta}+\frac{\delta_{\alpha \beta} U \Lambda}{M}, \\
\tilde{\mu}_{\mathrm{eff}} & =\epsilon_{0}-\frac{U}{M}\left|n_{0}+\Lambda\right| .
\end{aligned}
$$

We see that for $U \rightarrow 0$, the HFB approximation reduces to the Bogoliubov theory. This is due to the fact that the interaction is taken to be momentum-independent contact interaction: if this was not the case, $\Sigma_{11}$ and thus the diagonal blocks of $H_{\mathrm{HFB}}$ would acquire a more complicated momentum-dependent form. Therefore, the Bogoliubov theory gives the same results as HFB theory at the limit of $U \rightarrow 0$ even when the excitation density $n_{\mathrm{ex}}$ is comparable to the condensate density $n_{0}$, i.e., $n_{\mathrm{ex}} \sim n_{0}$.

Figure S2 in SM [71] compares both Bogoliubov and HFB methods in the case of the kagome flat-band condensate. In panels (a) and (b) we depict $c_{s}$ and in (d)-(f) $n_{\text {ex }}$ as a function of interaction for three different total densities. One can see that at the limit of $U \rightarrow 0$, the $n_{\mathrm{ex}}$ of the HFB method approaches the Bogoliubov result, consistent with our discussion in the preceding paragraph. We have confirmed that the HFB results of Fig. S2 are self-consistent and that their excitation spectra feature the gapless Goldstone mode at $\mathbf{k}_{c}$. We further note that utilizing the HFB method at total densities even smaller than those depicted in Fig. S2 does not yield self-consistent results, indicating that either the condensate becomes unstable or the HFB method breaks down in the small density limit.
[1] J. P. Provost and G. Vallee, Commun. Math. Phys. 76, 289 (1980).

[2] R. Resta, Eur. Phys. J. B 79, 121 (2011).

[3] G. Sundaram and Q. Niu, Phys. Rev. B 59, 14915 (1999).

[4] K. v. Klitzing, G. Dorda, and M. Pepper, Phys. Rev. Lett. 45, 494 (1980).

[5] D. J. Thouless, M. Kohmoto, M. P. Nightingale, and M. den Nijs, Phys. Rev. Lett. 49, 405 (1982).

[6] F. D. M. Haldane, Phys. Rev. Lett. 61, 2015 (1988).

[7] C. L. Kane and E. J. Mele, Phys. Rev. Lett. 95, 226801 (2005).

[8] M. Z. Hasan and C. L. Kane, Rev. Mod. Phys. 82, 3045 (2010).
[9] B. A. Bernevig and T. L. Hughes, Topological Insulators and Topological Superconductors (Princeton University Press, Princeton, NJ, 2013), p. 247.

[10] S. Peotta and P. Törmä, Nat. Commun. 6, 8944 (2015).

[11] A. Julku, S. Peotta, T. I. Vanhala, D.-H. Kim, and P. Törmä, Phys. Rev. Lett. 117, 045303 (2016).

[12] L. Liang, T. I. Vanhala, S. Peotta, T. Siro, A. Harju, and P. Törmä, Phys. Rev. B 95, 024515 (2017).

[13] Z. Wang, G. Chaudhary, Q. Chen, and K. Levin, Phys. Rev. B 102, 184504 (2020). 
[14] F. Piéchon, A. Raoux, J.-N. Fuchs, and G. Montambaux, Phys. Rev. B 94, 134423 (2016).

[15] J.-W. Rhim, K. Kim, and B.-J. Yang, Nature (London) 584, 59 (2020).

[16] G. E. Topp, C. J. Eckhardt, D. M. Kennes, M. A. Sentef, and P. Törmä, Phys. Rev. B 104, 064306 (2021).

[17] X. Hu, T. Hyart, D. I. Pikulin, and E. Rossi, Phys. Rev. Lett. 123, 237002 (2019).

[18] A. Julku, T. J. Peltonen, L. Liang, T. T. Heikkilä, and P. Törmä, Phys. Rev. B 101, 060505(R) (2020).

[19] F. Xie, Z. Song, B. Lian, and B. A. Bernevig, Phys. Rev. Lett 124, 167002 (2020).

[20] L. Classen, Physics 13, 23 (2020).

[21] F. D. M. Haldane and S. Raghu, Phys. Rev. Lett. 100, 013904 (2008).

[22] Z. Wang, Y. Chong, J. D. Joannopoulos, and M. Soljačić, Nature (London) 461, 772 (2009).

[23] H. Miyake, G. A. Siviloglou, C. J. Kennedy, W. C. Burton, and W. Ketterle, Phys. Rev. Lett. 111, 185302 (2013).

[24] M. Aidelsburger, M. Lohse, C. Schweizer, M. Atala, J. T. Barreiro, S. Nascimbene, N. R. Cooper, I. Bloch, and N. Goldman, Nat. Phys. 11, 162 (2015).

[25] S. Sugawa, F. Salces-Carcoba, A. R. Perry, Y. Yue, and I. B. Spielman, Science 360, 1429 (2018).

[26] L. Lu, J. D. Joannopoulos, and M. Soljačić, Nat. Photonics 8, 821 (2014).

[27] B. Bahari, B. Ndao, A. Vallini, A. El Amili, Y. Fainman, and B. Kante, Science 358, 636 (2017).

[28] A. B. Khanikaev and G. Shvets, Nat. Photonics 11, 763 (2017).

[29] T. Ozawa, H. M. Price, A. Amo, N. Goldman, M. Hafezi, L. Lu, M. C. Rechtsman, D. Schuster, J. Simon, O. Zilberberg, and I. Carusotto, Rev. Mod. Phys. 91, 015006 (2019).

[30] A. Gianfrate, O. Bleu, L. Dominici, V. Ardizzone, M. De Giorgi, D. Ballarini, G. Lerario, K. West, L. N. Pfeiffer, D. D. Solnyshkov, D. Sanvitto, and G. Malpuech, Nature (London) 578, 381 (2020).

[31] D. Pesin and L. Balents, Nat. Phys. 6, 376 (2010).

[32] T. Neupert, L. Santos, C. Chamon, and C. Mudry, Phys. Rev. Lett. 106, 236804 (2011).

[33] E. Tang, J.-W. Mei, and X.-G. Wen, Phys. Rev. Lett. 106, 236802 (2011).

[34] T. I. Vanhala, T. Siro, L. Liang, M. Troyer, A. Harju, and P. Törmä, Phys. Rev. Lett. 116, 225305 (2016).

[35] M. Sato and Y. Ando, Rep. Prog. Phys. 80, 076501 (2017).

[36] S. Rachel, Rep. Prog. Phys. 81, 116501 (2018).

[37] R. Roy, Phys. Rev. B 90, 165139 (2014).

[38] D. Leykam, A. Andreanov, and S. Flach, Adv. Phys.: X 3, 1473052 (2018).

[39] N. B. Kopnin, T. T. Heikkilä, and G. E. Volovik, Phys. Rev. B 83, 220503(R) (2011).

[40] S. Mukherjee, A. Spracklen, D. Choudhury, N. Goldman, P. Öhberg, E. Andersson, and R. R. Thomson, Phys. Rev. Lett. 114, 245504 (2015).

[41] S. Taie, H. Ozawa, T. Ichinose, T. Nishio, S. Nakajima, and Y. Takahashi, Science Adv. 1 (2015).

[42] R. A. Vicencio, C. Cantillano, L. Morales-Inostroza, B. Real, C. Mejía-Cortés, S. Weimann, A. Szameit, and M. I. Molina, Phys. Rev. Lett. 114, 245503 (2015).
[43] S. Kajiwara, Y. Urade, Y. Nakata, T. Nakanishi, and M. Kitano, Phys. Rev. B 93, 075126 (2016).

[44] T.-H. Leung, M. N. Schwarz, S.-W. Chang, C. D. Brown, G. Unnikrishnan, and D. Stamper-Kurn, Phys. Rev. Lett. 125, 133001 (2020).

[45] R. Drost, T. Ojanen, A. Harju, and P. Liljeroth, Nat. Phys. 13, 668 (2017).

[46] M. R. Slot, T. S. Gardenier, P. H. Jacobse, G. C. P. van Miert, S. N. Kempkes, S. J. M. Zevenhuizen, C. M. Smith, D. Vanmaekelbergh, and I. Swart, Nat. Phys. 13, 672 (2017).

[47] G. Li, A. Luican, J. M. B. Lopes dos Santos, A. H. Castro Neto, A. Reina, J. Kong, and E. Y. Andrei, Nat. Phys. 6, 109 (2010).

[48] Y. Cao, V. Fatemi, S. Fang, K. Watanabe, T. Taniguchi, E. Kaxiras, and P. Jarillo-Herrero, Nature (London) 556, 43 (2018).

[49] M. Yankowitz, S. Chen, H. Polshyn, Y. Zhang, K. Watanabe, T. Taniguchi, D. Graf, A. F. Young, and C. R. Dean, Science 363, 1059 (2019).

[50] J. Vidal, B. Douçot, R. Mosseri, and P. Butaud, Phys. Rev. Lett. 85, 3906 (2000).

[51] M. Di Liberto, S. Mukherjee, and N. Goldman, Phys. Rev. A 100, 043829 (2019).

[52] G. Gligorić, P. P. Beličev, D. Leykam, and A. Maluckov, Phys. Rev. A 99, 013826 (2019).

[53] C. Danieli, A. Andreanov, and S. Flach, Phys. Rev. B 102, 041116(R) (2020).

[54] S. Mukherjee, M. Di Liberto, P. Öhberg, R. R. Thomson, and N. Goldman, Phys. Rev. Lett. 121, 075502 (2018).

[55] V. Goblot, B. Rauer, F. Vicentini, A. Le Boité, E. Galopin, A. Lemaître, L. Le Gratiet, A. Harouri, I. Sagnes, S. Ravets, C. Ciuti, A. Amo, and J. Bloch, Phys. Rev. Lett. 123, 113901 (2019).

[56] S. D. Huber and E. Altman, Phys. Rev. B 82, 184502 (2010).

[57] F. Baboux, L. Ge, T. Jacqmin, M. Biondi, E. Galopin, A. Lemaître, L. Le Gratiet, I. Sagnes, S. Schmidt, H. E. Türeci, A. Amo, and J. Bloch, Phys. Rev. Lett. 116, 066402 (2016).

[58] C. E. Whittaker, E. Cancellieri, P. M. Walker, D. R. Gulevich, H. Schomerus, D. Vaitiekus, B. Royall, D. M. Whittaker, E. Clarke, I. V. Iorsh, I. A. Shelykh, M. S. Skolnick, and D. N. Krizhanovskii, Phys. Rev. Lett. 120, 097401 (2018).

[59] T. H. Harder, O. A. Egorov, J. Beierlein, P. Gagel, J. Michl, M. Emmerling, C. Schneider, U. Peschel, S. Höfling, and S. Klembt, Phys. Rev. B 102, 121302(R) (2020).

[60] F. Scafirimuto, D. Urbonas, M. A. Becker, U. Scherf, R. F. Mahrt, and T. Stöferle, Commun. Phys. 4, 39 (2021).

[61] T. H. Harder, O. A. Egorov, C. Krause, J. Beierlein, P. Gagel, M. Emmerling, C. Schneider, U. Peschel, S. Höfling, and S. Klembt, arXiv:2011.10766.

[62] Y.-Z. You, Z. Chen, X.-Q. Sun, and H. Zhai, Phys. Rev. Lett. 109, 265302 (2012).

[63] A. Fetter and J. Walecka, Quantum Theory of Many-Particle Systems, Dover Books on Physics Series (Dover, New York, 1971).

[64] A. Julku, G. M. Bruun, and P. Törmä, Phys. Rev. Lett. 127, 170404 (2021).

[65] L. Pitaevskii and S. Stringari, Bose-Einstein Condensation (Oxford University Press, Oxford, 2003).

[66] Y. Castin, in Coherent Atomic Matter Waves, edited by R. Caiser, C. Westbrook, and F. David (Springer, Berlin, 2001). 
[67] M. Berry, in Geometric Phases in Physics, edited by A. Shapere and F. Wilczek (World Scientific, Singapore, 1989).

[68] M. Iskin, Phys. B (Amsterdam) 592, 412260 (2020).

[69] J.-W. Rhim and B.-J. Yang, Phys. Rev. B 99, 045107 (2019).

[70] H. Shi and A. Griffin, Phys. Rep. 304, 1 (1998).

[71] See Supplemental Material at http://link.aps.org/supplemental/ 10.1103/PhysRevB.104.144507 for details on calculations of second-order correlations and superfluid weight as well as additional information on Hartree-Fock-Bogoliubov method.

[72] D. L. Bergman, C. Wu, and L. Balents, Phys. Rev. B 78, 125104 (2008).

[73] L. Yang, Phys. Rev. A 104, 023320 (2021).

[74] V. I. Yukalov, Phys. Part. Nuclei 42, 460 (2011). 